\title{
Communication in complex networks
}

\author{
Omar De la Cruz Cabrera ${ }^{\mathrm{a}}$, Jiafeng Jin ${ }^{\mathrm{a}}$, Silvia Noschese ${ }^{\mathrm{b}}$, Lothar Reichel $^{\mathrm{a}, *}$ \\ a Department of Mathematical Sciences, Kent State University, Kent, $\mathrm{OH} 44242$, USA \\ b Dipartimento di Matematica, SAPIENZA Università di Roma, P.le Aldo Moro 5, 00185 Roma, Italy
}

\section{A R T I C L E I N F O}

\section{Article history:}

Received 16 July 2021

Received in revised form 30 September 2021

Accepted 8 October 2021

Available online 13 October 2021

\section{Keywords:}

Network analysis

Sensitivity analysis

Krylov methods

\begin{abstract}
A B S T R A C T
One of the properties of interest in the analysis of networks is global communicability, i.e., how easy or difficult it is, generally, to reach nodes from other nodes by following edges. Different global communicability measures provide quantitative assessments of this property, emphasizing different aspects of the problem.

This paper investigates the sensitivity of global measures of communicability to local changes. In particular, for directed, weighted networks, we study how different global measures of communicability change when the weight of a single edge is changed; or, in the unweighted case, when an edge is added or removed. The measures we study include the total network communicability, based on the matrix exponential of the adjacency matrix, and the Perron network communicability, defined in terms of the Perron root of the adjacency matrix and the associated left and right eigenvectors.

Finding what local changes lead to the largest changes in global communicability has many potential applications, including assessing the resilience of a system to failure or attack, guidance for incremental system improvements, and studying the sensitivity of global communicability measures to errors in the network connection data.
\end{abstract}

(c) 2021 IMACS. Published by Elsevier B.V. All rights reserved.

\section{Introduction}

Many complex phenomena can be usefully modeled by networks. Mathematically, a network is represented by a graph, which consists of a set of vertices or nodes, and a set of edges that connect pairs of vertices. Network models often simplify the representation of a complex system by disregarding some minutiae of reality, to make it feasible to use mathematical and computational methods of analysis; see, e.g., [10,21] for many examples.

Sometimes, additional information about the vertices and/or edges is indispensable for a fuller and more realistic understanding of a complex system. Examples include the use of weighted networks [4,9,20], in which edges between vertices are assigned different numerical values, so-called "weights." In our setting, a higher weight for a given edge corresponds to a higher communication capacity between the nodes it connects.

An important characteristic of a network is how well communication can flow in it, i.e., how easy or difficult it is to reach one part of the network from another part by following edges. Several measures have been considered for quantifying communicability on a global scale. They include the diameter of the graph that represents the network, the average distance between nodes of this graph, and the communicability betweenness of nodes; see Estrada et al. [14]. Information transfer

\footnotetext{
* Corresponding author.

E-mail addresses: odelacru@kent.edu (O. De la Cruz Cabrera), jjin3@kent.edu (J. Jin), noschese@mat.uniroma1.it (S. Noschese), reichel@math.kent.edu (L. Reichel).
} 
between nodes also is studied with the aid of the thermal Green's function; here the network is considered submerged in a thermal bath of some temperature $T$; see Estrada et al. [11,13]. In this paper we concentrate on two communicability measures: the total network communicability, which was introduced by Benzi and Klymko [6], and the Perron network communicability, which we describe below.

This article explores the sensitivity of the communicability measures mentioned to small, local, changes of a network. Knowledge of the sensitivity can help answer several important questions about a network such as:

- How robust is a network to disturbances or attacks, and how can the network be modified to be more robust?

- Which edges of a network are very vulnerable, in the sense that the communicability decreases (relatively) significantly if these edges are removed?

- Can an addition of a new edge increase the communicability (relatively) significantly?

- Can a network be simplified by removing a few edges and retain essentially the same communicability?

- How sensitive is the measured communicability of a network to incomplete information about the existing edges?

The graphs we consider may be unweighted or weighted. In an unweighted graph all edges have the same weight, which we will choose to be one; in a weighted graph each edge has a positive weight. We are interested in which edge-weights to increase in order to increase the communicability of a graph the most, or which edges to add to or remove from a graph to achieve a significant increase or decrease, respectively, of the communicability. Our choice of which edge-weights to change, or which edges to add or remove, is based on the sensitivity of the communicability to changes in the edge-weight. We therefore investigate this sensitivity. Our approach is compared to some available approaches. Both undirected and directed graphs are considered.

We remark that graphs that have more than one connected component are deficient in their communicability, because not every node can communicate with every other node of the graph. Unless otherwise stated, we will assume the graphs under consideration to be connected. (For networks with more than one connected component, the results here can be applied separately to each component.)

This paper is organized as follows: Section 2 defines basic concepts about graphs. Notions of communicability are reviewed and a new one is introduced in Section 3. Computed illustrations for some small graphs are presented in Section 3.3. Section 4 describes numerical methods for estimating the sensitivity of the total network communicability, and the sensitivity of the Perron network communicability to changes in the weights, for large-scale networks. Section 5 presents a few computed results for large-scale networks. Concluding remarks are provided in Section 6.

\section{Basic definitions}

Networks are represented mathematically by graphs. The basic theory of graphs can be found in many textbooks; see, e.g., [10,21] for introductions focused on applications to the study of networks, and [7] for a deeper discussion of the matrices associated to a graph. Below we will briefly state the definitions we need in order to fix the notation.

A weighted graph $\mathcal{G}=\langle\mathcal{V}, \mathcal{E}, \mathcal{W}\rangle$ consists of a set of vertices or nodes $\mathcal{V}=\left\{v_{1}, v_{2}, \ldots, v_{n}\right\}$, a set of edges $\mathcal{E}=$ $\left\{e_{1}, e_{2}, \ldots, e_{m}\right\}$ that connect the nodes, and a map $\mathcal{W}$ that assigns to each edge a weight, which for the purposes of this article always will be a positive real number. An edge $e_{k}$ is said to be directed if it starts at a vertex $v_{i}$ and ends at a vertex $v_{j}$. This edge is denoted by $e\left(v_{i} \rightarrow v_{j}\right)$ and has the associated weight $w_{i j}>0$. If there also is an edge $e\left(v_{j} \rightarrow v_{i}\right)$ with the same weight $w_{j i}=w_{i j}$, then we may identify the directed edges $e\left(v_{i} \rightarrow v_{j}\right)$ and $e\left(v_{j} \rightarrow v_{i}\right)$ with an undirected edge with weight $w_{i j}$; we denote undirected edges by $e\left(v_{i} \leftrightarrow v_{j}\right)$. A graph with only undirected edges is said to be undirected; otherwise the graph is directed. In this work, we will consider only graphs without multiple edges or self-loops. The adjacency matrix for a graph $\mathcal{G}$ is the matrix $A=\left[w_{i j}\right]_{i, j=1}^{n} \in \mathbb{R}^{n \times n}$, whose entries are the edge-weights; if there is no edge $e\left(v_{i} \rightarrow v_{j}\right)$ in $\mathcal{G}$, then $w_{i j}=0$. For an unweighted graph, all positive entries $w_{i j}$ of $A$ equal one. When $\mathcal{G}$ is undirected, then $A$ is symmetric.

A sequence of edges (not necessarily distinct) such that $\left\{e\left(v_{1} \rightarrow v_{2}\right), e\left(v_{2} \rightarrow v_{3}\right), \ldots, e\left(v_{k} \rightarrow v_{k+1}\right)\right\}$ form a walk of length $k$. If $v_{k+1}=v_{1}$, then the walk is said to be closed. For further discussions on networks and graphs; see [10,21].

\section{Notions of communicability}

There are many measures of communicability of a network. For instance, the diameter of the graph that represents a network provides a measure of how easy it is for the nodes of the graph to communicate. We recall that for an unweighted graph, its diameter is the maximal length of the shortest path between any pair of distinct nodes of the graph. The definition has to be adjusted for weighted graphs. We will not use the diameter in this paper, because as a "worst case" measure it is fairly crude. For instance, let $\mathcal{G}$ be an unweighted complete graph with $n \geq 4$ nodes from which one edge is removed. Then the diameter of $\mathcal{G}$ is 2 . More edges can be removed so that the diameter remains 2 . In fact, one can remove any 1 or 2 edges from a complete graph with 4 nodes and then obtains a graph with diameter 2 .

This paper focuses on the total network communicability, which is defined with the aid of the exponential of the adjacency matrix, and on the Perron network communicability, which is defined with the Perron root and the right and left Perron vectors of the adjacency matrix. This section discusses these communicability measures and their sensitivity to 
changes in the weights that define the adjacency matrix. Small examples that illustrate the performance of these measures are presented.

\subsection{The modified matrix exponential and network communicability}

Consider an unweighted graph $\mathcal{G}$ with adjacency matrix $A \in \mathbb{R}^{n \times n}$. Then the $(i j)^{t h}$ entry of the matrix $A^{k}$ counts the number of walks of length $k$ between the vertices $v_{i}$ and $v_{j}$. For weighted graphs, the interpretation of the $(i j)^{t h}$ entry of the matrix $A^{k}$ has to be modified. A matrix function that is analytic at the origin and vanishes there can be defined by a formal Maclaurin series

$$
f(A)=\sum_{k=1}^{\infty} c_{k} A^{k}
$$

For the moment we ignore the convergence properties of this series. Long walks are usually considered less important than short walks, because information flows more easily through short walks than through long ones. Therefore, matrix functions applied in network analysis generally have the property that $0 \leq c_{k+1} \leq c_{k}$ for all sufficiently large $k$. The possibly most common matrix function used in network analysis is the matrix exponential; see [10,15] for discussions and illustrations. We prefer to use the modified matrix exponential

$$
\exp _{0}(A):=\exp (A)-I
$$

where $I$ denotes the identity matrix, because the first term in the Maclaurin series of $\exp (A)$ has no natural interpretation in the context of network modeling. For the modified matrix exponential, we have $c_{k}=1 / k$ ! for $k \geq 1$, and the series ( 1 ) converges for any adjacency matrix $A$. The quantity $\left[\exp _{0}(A)\right]_{i i}$ is commonly referred to as the subgraph centrality of the vertex $v_{i}$; it measures the ease of leaving node $v_{i}$ and returning to this node by following the edges of the graph; see $[10,15]$, though we remark that these references apply the matrix exponential instead of (2). The subgraph centrality is an appropriate measure for undirected graphs; a discussion about directed graphs is provided in [8].

The communicability between distinct vertices $v_{i}$ and $v_{j}, i \neq j$, is defined by

$$
\left[\exp _{0}(A)\right]_{i j}=\sum_{k=1}^{\infty} \frac{\left[A^{k}\right]_{i j}}{k !} ;
$$

see $[10,12]$ for the analogous definition based on $\exp (A)$. It accounts for all the possible routes of communication between the vertices $v_{i}$ and $v_{j}$ in the network defined by the adjacency matrix $A$, and assigns more weight to shorter routes than to longer ones. The larger the value of $\left[\exp _{0}(A)\right]_{i j}$, the better is the communicability between the vertices $v_{i}$ and $v_{j}$.

We measure how effectively information can be transmitted across the whole network by the total network communicability,

$$
C^{\mathrm{TN}}(A)=\mathbf{1}^{T} \exp _{0}(A) \mathbf{1}
$$

where $\mathbf{1}=[1,1, \ldots, 1]^{T} \in \mathbb{R}^{n}$. Benzi and Klymko [6] defined the total network communicability with exp $(A)$; this yields values that are $n$ larger than the values obtained with (3).

We are interested in determining the sensitivity of the total network communicability to changes in the weight $w_{i j}$ of the edge $e\left(v_{i} \rightarrow v_{j}\right)$. Therefore, we compute the partial derivative of $C^{\mathrm{TN}}(A)$ with respect to $w_{i j}$. For that, we introduce the Fréchet derivative $L\left(A, E_{i j}\right)$ of the modified matrix exponential $\exp _{0}(A)$ with respect to the direction $E_{i j}=\mathbf{e}_{i} \mathbf{e}_{j}^{T}$, where $\mathbf{e}_{k}=[0, \ldots, 0,1,0, \ldots, 0]^{T} \in \mathbb{R}^{n}$ denotes the $k$ th axis vector, given by

$$
L\left(A, E_{i j}\right)=\lim _{t \rightarrow 0} \frac{\exp _{0}\left(A+t E_{i j}\right)-\exp _{0}(A)}{t}
$$

see, e.g., [16,22]. Then

$$
\frac{\partial C^{\mathrm{TN}}(A)}{\partial w_{i j}}=\lim _{t \rightarrow 0} \frac{C^{\mathrm{TN}}\left(A+t E_{i j}\right)-C^{\mathrm{TN}}(A)}{t}=\mathbf{1}^{T} \cdot L\left(A, E_{i j}\right) \cdot \mathbf{1}
$$

shows the rate of change of the total network communicability between the vertices $v_{i}$ and $v_{j}$ in direction $E_{i j}$ due to a change in the edge-weight $w_{i j}$.

Definition 1. Let $\mathcal{G}=\{\mathcal{V}, \mathcal{E}, \mathcal{W}\}$ be a graph with adjacency matrix $A=\left[w_{i j}\right] \in \mathbb{R}^{n \times n}$, where $w_{i j}>0$ if there is an edge $e\left(v_{i} \rightarrow v_{j}\right)$ in $\mathcal{G}$, and $w_{i j}=0$ otherwise. We define the total network sensitivity with respect to the weight $w_{i j}$ as

$$
S_{i j}^{\mathrm{TN}}(A)=\mathbf{1}^{T} \cdot L\left(A, E_{i j}\right) \cdot \mathbf{1}
$$


as well as the total network sensitivity as

$$
S^{\mathrm{TN}}(A)=\sum_{i, j=1}^{n} S_{i j}^{\mathrm{TN}}(A) \text {. }
$$

The total network sensitivity with respect to the weight $w_{i j}$ shows the rate of change of $C^{\mathrm{TN}}(A)$ with respect to a change in the edge-weight $w_{i j}$. The following result follows from Higham [16, Theorem 3.6].

Proposition 1. Let $f$ be $2 n-1$ times continuously differentiable in a connected open set $\Omega$ in the complex plane containing the origin, and assume that the spectrum of the matrix $A \in \mathbb{R}^{n \times n}$ is in $\Omega$. Then the Fréchet derivative $L\left(A, E_{i j}\right)$ exists and satisfies

$$
f\left(\left[\begin{array}{cc}
A & E_{i j} \\
0 & A
\end{array}\right]\right)=\left[\begin{array}{cc}
f(A) & L\left(A, E_{i j}\right) \\
0 & f(A)
\end{array}\right] .
$$

We are interested in the special case of Proposition 1 when $f(t)=\exp (t)-1$. Then the Fréchet derivative $L\left(A, E_{i j}\right)$ in (6) is defined by (4). One has

$$
\exp _{0}\left(A+t E_{i j}\right)-\exp _{0}(A)-t L\left(A, E_{i j}\right)=O\left(t^{2}\right) \text { as } t \rightarrow 0 .
$$

\subsection{Perron network communicability}

Let $A=\left[w_{i j}\right] \in \mathbb{R}^{n \times n}$ be a nonnegative irreducible adjacency matrix for a graph and let $\rho$ be its Perron root (see, e.g., [17] for a full treatment of the Perron-Frobenius Theorem). Then there are unique right and left real eigenvectors $\mathbf{x}=$ $\left[x_{1}, x_{2}, \ldots, x_{n}\right]^{T} \in \mathbb{R}^{n}$ and $\mathbf{y}=\left[y_{1}, y_{2}, \ldots, y_{n}\right]^{T} \in \mathbb{R}^{n}$, respectively, of unit Euclidean norm with positive entries associated with $\rho$, i.e.,

$$
A \mathbf{x}=\rho \mathbf{x}, \quad \mathbf{y}^{T} A=\rho \mathbf{y}^{T} .
$$

We recall that a node $v_{i}$ is referred to as a sink if there are no edges pointing from this node to any other node, and a node $v_{i}$ is said to be a source when there are no edges from other nodes pointing to $v_{i}$. Undirected edges should be considered as "two-way streets" in this context. A directed graph is said to be strongly connected if every vertex $v_{i}$ can be reached from any other vertex $v_{j}, j \neq i$, by following edges in their direction. It is well known that an adjacency matrix is irreducible if and only if the associated graph is strongly connected; see, e.g., [17]. In particular, a graph with a sink or source is not strongly connected, and the adjacency matrix $A$ for such a graph is reducible. A common approach to obtain a nearby irreducible adjacency matrix is to make a small positive perturbation of every entry of $A$. For instance, we may replace $A$ by the irreducible adjacency matrix $\hat{A}=A+\delta A \in \mathbb{R}^{n \times n}$, where $\delta A$ is a small multiple of the matrix $\mathbf{1 1}^{T}$ and $\mathbf{1}=[1,1, \ldots, 1]^{T} \in \mathbb{R}^{n}$. This makes the matrix $\hat{A}=A+\delta A$ irreducible with $\|\hat{A}\|_{2} \approx\|A\|_{2}$. Here and throughout this paper, $\|\cdot\|_{2}$ denotes the spectral matrix norm or the Euclidean vector norm. In this section we will assume that, in case the given adjacency matrix $A$ is reducible, it is modified in this manner. We may therefore assume that the right and left Perron vectors are unique up to scaling.

Let $C \in \mathbb{R}^{n \times n}$ be a nonnegative matrix such that $\|C\|_{2}=1$, and let $\varepsilon>0$ be small. Denote the Perron root of $A+\varepsilon C$ by $\rho+\delta \rho$. Then

$$
\delta \rho=\varepsilon \frac{\mathbf{y}^{T} C \mathbf{x}}{\mathbf{y}^{T} \mathbf{x}}+O\left(\varepsilon^{2}\right) ;
$$

see [19]. Moreover,

$$
\frac{\mathbf{y}^{T} C \mathbf{x}}{\mathbf{y}^{T} \mathbf{x}}=\frac{\left|\mathbf{y}^{T} C \mathbf{x}\right|}{\mathbf{y}^{T} \mathbf{x}} \leq \frac{\|\mathbf{y}\|_{2}\|C\|_{2}\|\mathbf{x}\|_{2}}{\mathbf{y}^{T} \mathbf{x}}=\frac{1}{\cos \theta},
$$

where $\theta$ is the angle between $\mathbf{x}$ and $\mathbf{y}$. The quantity $1 / \cos \theta$ is referred to as the condition number of $\rho$ and denoted by $\kappa(\rho)$; see Wilkinson [28, Chapter 2]. Equality is attained in (9) for $C=\mathbf{y x}^{T}$.

Consider increasing the entry $w_{i j}, i \neq j$, of $A$ slightly by $\varepsilon>0$. This corresponds to increasing the weight $w_{i j}$ of an existing edge $e\left(v_{i} \rightarrow v_{j}\right)$ by $\varepsilon$, or to introducing a new edge $e\left(v_{i} \rightarrow v_{j}\right)$ with weight $\varepsilon$. The corresponding matrix $C$ is given by

$$
C=E_{i j}=\mathbf{e}_{i} \mathbf{e}_{j}^{T}
$$

The impact on the Perron root of the change $\varepsilon C$ of $A$ is

$$
\delta \rho=\varepsilon \frac{y_{i} x_{j}}{\mathbf{y}^{T} \mathbf{x}}+O\left(\varepsilon^{2}\right) .
$$


We are interested in choosing a perturbation $\varepsilon C$ of $A$ with $C$ of the form (10), so that $\rho$ is increased as much as possible. The above relation shows that we should choose $i$ and $j$ so that

$$
x_{j}=\max _{1 \leq k \leq n} x_{k}, \quad y_{i}=\max _{1 \leq k \leq n} y_{k}
$$

Recall that $\mathbf{x}$ and $\mathbf{y}$ are unit vectors. Therefore, $x_{j}=1$ and $y_{i}=1$ implies that $x_{k}=0$ for all $k \neq j$ and $y_{k}=0$ for all $k \neq i$. Then $C=E_{i j}=\mathbf{y} \mathbf{x}^{T}$ and the maximum perturbation of the Perron root is $\delta \rho=\varepsilon \kappa(\rho)$.

We define the Perron root sensitivity with respect to the direction $E_{i j}=\mathbf{e}_{i} \mathbf{e}_{j}^{T}$ as

$$
S_{i j}^{\mathrm{PR}}(A)=\frac{y_{i} x_{j}}{\mathbf{y}^{T} \mathbf{x}},
$$

as well as the Perron root sensitivity matrix

$$
S^{\mathrm{PR}}(A)=\left[S_{i j}^{\mathrm{PR}}(A)\right]_{i, j=1}^{n}=\frac{\mathbf{y x}^{T}}{\mathbf{y}^{T} \mathbf{x}} \in \mathbb{R}^{n \times n}
$$

Finally, we introduce the Perron network communicability,

$$
C^{\mathrm{PN}}(A)=\exp _{0}(\rho) \mathbf{1}^{T} \mathbf{x y}^{T} \mathbf{1}=\exp _{0}(\rho)\left(\sum_{j=1}^{n} x_{j}\right)\left(\sum_{j=1}^{n} y_{j}\right),
$$

which is analogous to the total network communicability (3), but is easier to compute for a large adjacency matrix $A$. Let $\|\cdot\|_{1}$ denote the vector 1 -norm. Since

$$
\sum_{j=1}^{n} x_{j}=\|\mathbf{x}\|_{1} \leq n^{1 / 2}\|\mathbf{x}\|_{2}=n^{1 / 2}, \quad \sum_{j=1}^{n} y_{j}=\|\mathbf{y}\|_{1} \leq n^{1 / 2}\|\mathbf{y}\|_{2}=n^{1 / 2},
$$

we have the bound

$$
C^{\mathrm{PN}}(A) \leq n \exp _{0}(\rho)
$$

In general, we expect the Perron network communicability to increase the most when increasing the edge-weight that makes the Perron root change the most.

An alternative way to study the sensitivity of the Perron network communicability is to determine the first-order partial derivative of $C^{\mathrm{PN}}(A)$ with respect to $w_{i j}$. Introduce the Fréchet derivative $L^{\mathrm{PN}}\left(A, E_{i j}\right)$ of the matrix function $\exp _{0}(\rho) \mathbf{x y}^{T}$ with respect to the direction $E_{i j}=\mathbf{e}_{i} \mathbf{e}_{j}^{T}$, i.e.,

$$
L^{\mathrm{PN}}\left(A, E_{i j}\right)=\lim _{t \rightarrow 0} \frac{\exp _{0}(\hat{\rho}(t)) \hat{\mathbf{x}}(t) \hat{\mathbf{y}}(t)^{T}-\exp _{0}(\rho) \mathbf{x y}^{T}}{t},
$$

where $\rho, \mathbf{x}$, and $\mathbf{y}$ are the Perron root, and the right and left Perron vectors of $A$, respectively, and $\hat{\rho}(t), \hat{\mathbf{x}}(t)$, and $\hat{\mathbf{y}}(t)$ are the Perron root, and the right and left Perron vectors of $A+t E_{i j}$. Then

$$
\frac{\partial C^{\mathrm{PN}}(A)}{\partial w_{i j}}=\lim _{t \rightarrow 0} \frac{C^{\mathrm{PN}}\left(A+t E_{i j}\right)-C^{\mathrm{PN}}(A)}{t}=\mathbf{1}^{T} \cdot L^{\mathrm{PN}}\left(A, E_{i j}\right) \cdot \mathbf{1}
$$

is the rate of change of the Perron network communicability between the vertices $v_{i}$ and $v_{j}$ in the direction $E_{i j}$ due to a change in $w_{i j}$. For the examples shown in the following sections, we choose $t=2 \cdot 10^{-5}$ when computing $L^{\mathrm{PN}}\left(A, E_{i j}\right)$ unless explicitly stated otherwise.

Definition 2. Let $\mathcal{G}=\{\mathcal{V}, \mathcal{E}, \mathcal{W}\}$ be a graph with adjacency matrix $A=\left[w_{i j}\right] \in \mathbb{R}^{n \times n}$, where $w_{i j}>0$ if there is an edge $e\left(v_{i} \rightarrow v_{j}\right)$ in $\mathcal{G}$, and $w_{i j}=0$ otherwise. We define the Perron network sensitivity with respect to the weight $w_{i j}$ as

$$
S_{i j}^{\mathrm{PN}}(A)=\mathbf{1}^{T} \cdot L^{\mathrm{PN}}\left(A, E_{i j}\right) \cdot \mathbf{1},
$$

as well as the Perron network sensitivity as

$$
S^{\mathrm{PN}}(A)=\sum_{i, j=1}^{n} S_{i j}^{\mathrm{PN}}(A) .
$$


The Perron network sensitivity with respect to the weight $w_{i j}$ shows the rate of change of $C^{\mathrm{PN}}(A)$ with respect to a change in the edge-weight $w_{i j}$. We are interested in investigating how $C^{\mathrm{PN}}(A)$ relates to $C^{\mathrm{TN}}(A)$. Assume first that the spectral factorizations

$$
A=X \Lambda X^{-1}, \quad A^{T}=\widetilde{Y} \Lambda \widetilde{Y}^{-1}
$$

exist, where $\Lambda=\operatorname{diag}\left[\rho, \lambda_{2}, \ldots, \lambda_{n}\right]$ and the columns of the eigenvector matrix $X=\left[\mathbf{x}, \mathbf{x}_{2}, \ldots, \mathbf{x}_{n}\right]$ are scaled to be of unit Euclidean norm. We remark that matrices with a spectral factorization are dense among all matrices in $\mathbb{R}^{n \times n}$. We may choose $\widetilde{Y}=\left[\widetilde{\mathbf{y}}, \widetilde{\mathbf{y}}_{2}, \ldots, \widetilde{\mathbf{y}}_{n}\right]=X^{-T}$. Then $A=X \Lambda \widetilde{Y}^{T}$. The vector $\widetilde{\mathbf{y}}$ is a rescaling of the left Perron vector $\mathbf{y}$ in (8). This normalization of the columns of $\tilde{Y}$ yields $\widetilde{\mathbf{y}}^{T} \mathbf{x}=1$ and $\widetilde{\mathbf{y}}_{j}^{T} \mathbf{x}_{j}=1$ for $j=2,3, \ldots, n$. In particular, this implies

$$
1=\widetilde{\mathbf{y}}^{T} \mathbf{x}=\|\mathbf{x}\|\|\widetilde{\mathbf{y}}\| \cos \theta, \quad \text { i.e., } \quad\|\widetilde{\mathbf{y}}\|=\frac{1}{\cos \theta}=\kappa(\rho) .
$$

Assume that $\rho$ is significantly larger than $\left|\lambda_{j}\right|$ for $j=2,3, \ldots, n$. Then

$$
\begin{aligned}
C^{\mathrm{TN}}(A) & =\mathbf{1}^{T} X \exp _{0}(\Lambda) \widetilde{Y}^{T} \mathbf{1} \\
& =\exp _{0}(\rho) \mathbf{1}^{T} \tilde{\mathbf{x}}^{T} \mathbf{1}+\sum_{j=2}^{n} \exp _{0}\left(\lambda_{j}\right) \mathbf{1}^{T} \mathbf{x}_{j} \widetilde{\mathbf{y}}_{j}^{T} \mathbf{1} \\
& =\kappa(\rho) C^{\mathrm{PN}}(A)+\sum_{j=2}^{n} \exp _{0}\left(\lambda_{j}\right) \mathbf{1}^{T} \mathbf{x}_{j} \widetilde{\mathbf{y}}_{j}^{T} \mathbf{1} \\
& \approx \kappa(\rho) C^{\mathrm{PN}}(A) .
\end{aligned}
$$

Thus, the total network communicability depends on the conditioning of the Perron root. For a general matrix $A$, we can use the bounds $\left|\mathbf{1}^{T} \mathbf{x}_{j}\right| \leq n^{1 / 2}$ and $\left|\widetilde{\mathbf{y}}_{j}^{T} \mathbf{1}\right| \leq\left\|\widetilde{\mathbf{y}}_{j}\right\|_{1}$ in (14). When the graph that defines $A$ is undirected, the matrix $A$ is symmetric, and we can let $\widetilde{Y}=X$ be orthogonal. In this case, we have $\kappa(\rho)=1$ and can use

$$
\left|\mathbf{1}^{T} \mathbf{x}_{j} \widetilde{\mathbf{y}}_{j}^{T} \mathbf{1}\right| \leq n, \quad j=2,3, \ldots, n,
$$

in (14).

If the matrix $A$ does not have the factorizations (13), then an analogous argument can be made using the Jordan canonical form, where we use the fact that the right and left Perron vectors exist and are unique up to scaling also in this situation.

We finally remark that both the computation of the total network sensitivity $S^{\mathrm{TN}}(A)$ and of the Perron network sensitivity $S^{\mathrm{PN}}(A)$ for a graph with $n$ nodes requires the evaluation of $n^{2}$ Fréchet derivatives. This makes the evaluation of these quantities expensive for networks with many nodes. The evaluation of the Perron root sensitivity matrix $S^{\mathrm{PR}}(A)$ typically is much cheaper for large networks. We will return to this issue below.

\subsection{Examples with small networks}

This section describes a few small examples that illustrate the use of Fréchet derivatives and the Perron root sensitivity. The computational effort required to compute all Fréchet derivatives for large-scale networks can be significant. How to reduce the computational effort for large-scale networks is discussed in Section 4.

In the first example, which is a small weighted graph, we compute the total network sensitivity and the Perron network sensitivity with respect to the weights $w_{i j}$ for $i \neq j$ to decide which edge-weight should be increased to enhance the total network communicability or the Perron network communicability as much as possible. We study the Perron root sensitivity by computing the left and right Perron vectors of $A$. The latter computations suggest which weight should be increased. Our second example differs from the first one in that the graph is unweighted and directed.

Example 3.1. Consider the weighted graph of Fig. 1 with associated adjacency matrix

$$
A=\left[\begin{array}{llll}
0 & 2 & 4 & 2 \\
5 & 0 & 1 & 1 \\
5 & 4 & 0 & 5 \\
3 & 4 & 3 & 0
\end{array}\right]
$$

The corresponding Perron root sensitivity matrix of $A$ is 


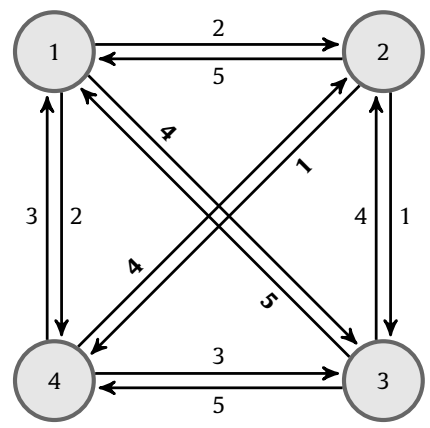

Fig. 1. Graph of Example 3.1. The edge-weights are marked next to the edges.

Table 1

Example 3.1: The total network sensitivity $S_{i j}^{\mathrm{TN}}$ and the Perron network sensitivity $S_{i j}^{\mathrm{PN}}$ with respect to changes in the weight $w_{i j}$, for $i \neq j$, along with the total network communicability and the Perron network communicability when the weight $w_{i j}$ of the edge $e\left(v_{i} \rightarrow v_{j}\right)$ is increased by one.

\begin{tabular}{llllll}
\hline$\{i, j\}$ & $S_{i j}^{\mathrm{TN}}$ & $C^{\mathrm{TN}}\left(A+E_{i j}\right)$ & $\{i, j\}$ & $S_{i j}^{\mathrm{PN}}$ & $C^{\mathrm{PN}}\left(A+E_{i j}\right)$ \\
\hline$\{1,3\}$ & 22615 & 82269 & $\{1,3\}$ & 22781 & 79872 \\
$\{2,3\}$ & 18221 & 76339 & $\{2,3\}$ & 18247 & 73711 \\
$\{1,4\}$ & 17662 & 75511 & $\{1,4\}$ & 17577 & 72722 \\
$\{4,3\}$ & 15611 & 73124 & $\{4,3\}$ & 15009 & 69720 \\
$\{2,4\}$ & 14225 & 71324 & $\{2,4\}$ & 14078 & 68481 \\
$\{1,2\}$ & 13151 & 70097 & $\{1,2\}$ & 12411 & 66543 \\
$\{2,1\}$ & 12957 & 69606 & $\{2,1\}$ & 12394 & 66250 \\
$\{3,4\}$ & 12883 & 69588 & $\{3,4\}$ & 12134 & 66022 \\
$\{3,1\}$ & 11734 & 68303 & $\{3,1\}$ & 10666 & 64389 \\
$\{4,1\}$ & 11098 & 67434 & $\{4,1\}$ & 10188 & 63702 \\
$\{3,2\}$ & 9585 & 65627 & $\{3,2\}$ & 8562 & 61789 \\
$\{4,2\}$ & 9063 & 65011 & $\{4,2\}$ & 8176 & 61329 \\
\hline
\end{tabular}

$$
S^{\mathrm{PR}}(A)=\left[\begin{array}{llll}
0.2956 & 0.2339 & 0.4241 & 0.3250 \\
0.2336 & 0.1848 & 0.3352 & 0.2568 \\
0.2109 & 0.1669 & 0.3026 & 0.2319 \\
0.1973 & 0.1562 & 0.2832 & 0.2170
\end{array}\right],
$$

which shows that the Perron root sensitivity $S_{i j}^{\mathrm{PR}}$ is maximized for $\{i, j\}=\{1,3\}$. This indicates that the Perron root is increased the most when increasing the weight $w_{13}$ of the edge $e\left(v_{1} \rightarrow v_{3}\right)$. We expect the Perron network communicability to increase the most when increasing the edge-weight that makes the Perron root change the most. Table 1 confirms this. The table displays the total network sensitivity and the Perron network sensitivity with respect to changes in the weights $w_{i j}$ for $i \neq j$, as well as the total network communicability and the Perron network communicability of the graph obtained when increasing each weight $w_{i j}, i \neq j$, by one. The table shows the total network communicability to increase the most by increasing the weight of the edge with the largest total network sensitivity $S_{i j}^{\mathrm{TN}}$, i.e., weight $w_{13}$. Increasing this weight, which also is associated with the largest Perron network sensitivity $S_{13}^{\mathrm{PN}}$, gives the largest Perron network communicability, $C^{\mathrm{PN}}\left(A+E_{13}\right)$. Thus, if the edges represent roads, the weights represent the width of each road, and we would like to increase the communicability the most by widening one road, then we should widen the road represented by the edge $e\left(v_{1} \rightarrow v_{3}\right)$.

The total network sensitivities $S_{i j}^{\mathrm{TN}}$ and the Perron network sensitivities $S_{i j}^{\mathrm{PN}}$ with respect to changes in the weights $w_{i j}$ of Table 1 also can be used to assess which weight(s) to decrease to reduce the total network communicability or the Perron network communicability of the network of Fig. 1 the most. The fact that $S_{13}^{\mathrm{TN}}$ and $S_{13}^{\mathrm{PN}}$ are the largest sensitivities suggests that we should reduce the weight $w_{13}$ to reduce the total network communicability and the Perron network communicability the most. Furthermore, the Perron root sensitivity matrix (16) suggests that both the Perron network communicability (11) and the Perron root will decrease the most when decreasing the weight $w_{13}$. Indeed, tabulating $C^{\mathrm{TN}}\left(A-E_{i j}\right)$ and $C^{\mathrm{PN}}\left(A-E_{i j}\right)$ for $1 \leq i, j \leq 4, i \neq j$, shows $C^{\mathrm{TN}}\left(A-E_{13}\right)$ and $C^{\mathrm{PN}}\left(A-E_{13}\right)$ to be minimal.

In the adjacency matrix (15) all off-diagonal entries are positive. This is not important for the approach described. We can compute the total network sensitivity (5) and the Perron network sensitivity (12) independently of the values of the weights $w_{i j}$. If $S_{i j}^{\mathrm{TN}}$ or $S_{i j}^{\mathrm{PN}}$ is the largest sensitivity and $w_{i j}=0$, then this indicates that the total network communicability or the Perron network communicability may be increased the most by adding the edge $e\left(v_{i} \rightarrow v_{j}\right)$ to the graph. 


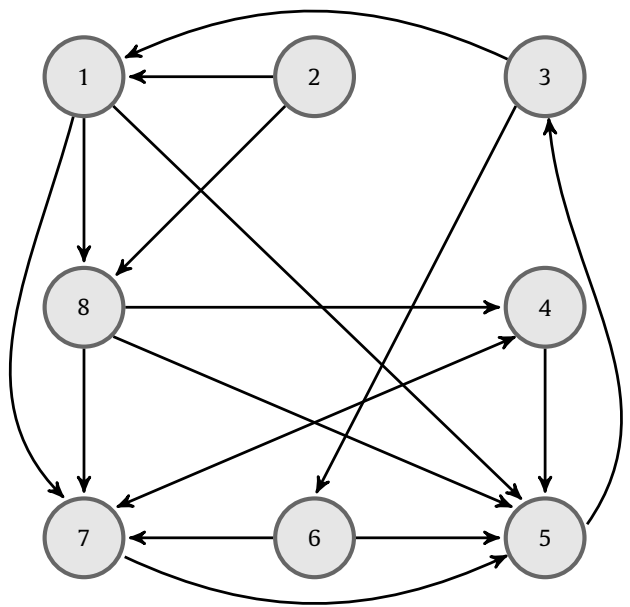

Fig. 2. Graph of Example 3.2. All edges have unit weight.

Table 2

Example 3.2: The five largest total network sensitivities $S_{i j}^{\mathrm{TN}}$ and Perron network sensitivities $S_{i j}^{\mathrm{PN}}$ with respect to perturbations in the weights $w_{i j}$, for $i \neq j$, along with the corresponding total network communicabilities and Perron network communicabilities when the edge-weight $w_{i j}$ is increased by one.

\begin{tabular}{llllll}
\hline$\{i, j\}$ & $S_{i j}^{\mathrm{TN}}$ & $C^{\mathrm{TN}}\left(A+E_{i j}\right)$ & $\{i, j\}$ & $S_{i j}^{\mathrm{PN}}$ & $C^{\mathrm{PN}}\left(\hat{A}+E_{i j}\right)$ \\
\hline$\{5,1\}$ & 16.8311 & 68.1499 & $\{5,2\}$ & 28.5369 & 58.3264 \\
$\{5,8\}$ & 16.0552 & 67.3050 & $\{5,1\}$ & 23.0099 & 56.9987 \\
$\{5,2\}$ & 14.9415 & 65.2404 & $\{5,3\}$ & 19.9219 & 51.2350 \\
$\{5,3\}$ & 14.1656 & 64.4248 & $\{5,8\}$ & 19.5778 & 53.8728 \\
$\{7,1\}$ & 13.2831 & 64.1815 & $\{7,2\}$ & 18.2576 & 50.5741 \\
\hline
\end{tabular}

Example 3.2. Let $A \in \mathbb{R}^{8 \times 8}$ be the adjacency matrix for the unweighted directed graph of Fig. 2 . All its entries are either one of zero. The graph is not strongly connected, and therefore the matrix $A$ is reducible. To obtain an irreducible matrix $\hat{A}$, we add the perturbation matrix $\delta A=\delta \cdot \mathbf{1 1}^{T}$ to $A$ for some $\delta>0$; thus, $\hat{A}=A+\delta A$. We choose the value of $\delta$ as follows: Compute the Perron vectors for $\delta=10^{-4}$ and then reduce $\delta$ by a factor 10 and determine new Perron vectors until the edge determined for two consecutive $\delta$-values is the same. For the present example, this gives $\delta=10^{-5}$. The matrix $\hat{A}=A+10^{-5} \cdot \mathbf{1 1}^{T}$ so determined is irreducible and the right and left Perron vectors are unique up to scaling. We find the three largest entries of the Perron root sensitivity matrix $S^{\mathrm{PR}}(\hat{A})$ of $\hat{A}$ to be

$$
S^{\mathrm{PR}}(\hat{A})_{5,1}=0.477305, \quad S^{\mathrm{PR}}(\hat{A})_{5,2}=0.477298, \quad S^{\mathrm{PR}}(\hat{A})_{5,8}=0.400601 .
$$

This suggests that the Perron root may be increased the most by inserting the edge $e\left(v_{5} \rightarrow v_{1}\right)$ into the graph. Typically, the Perron network communicability is increased the most by increasing the weight for an edge (or inserting an edge) that results in the largest increase of the Perron root. However, as is illustrated by Table 2, this might not be the case when the largest Perron root sensitivities $S^{\mathrm{PR}}(\hat{A})_{i, j}$ are very close in size as in the present example, where $S_{5,1}^{\mathrm{PR}}$ and $S_{5,2}^{\mathrm{PR}}$ are very close. The table shows the Perron network communicability to increase the most by adding the edge $e\left(v_{5} \rightarrow v_{2}\right)$. Table 2 shows the top five total network sensitivities and Perron network sensitivities with respect to perturbations in $w_{i j}$ for $i \neq j$, along with the total network communicabilities and Perron network communicabilities of the graph obtained when increasing each edge-weight $w_{i j}$ by one. The table shows the total network communicability to increase the most when adding the edge associated with the largest total network sensitivity $S_{i j}^{\mathrm{TN}}$. The Perron network communicability increases the most by inserting the edge associated with the largest Perron network sensitivity $S_{i j}^{\mathrm{PN}}$.

For some graphs one can prove where to add an additional edge to maximize the total network sensitivity. The following result provides an illustration.

Theorem 1. Let $A=\left[w_{i j}\right] \in \mathbb{R}^{n \times n}$ be an adjacency matrix with all superdiagonal entries equal to one and all other entries equal to zero. Fig. 3 displays the associated graph for $n=8$. Let $S_{i j}^{\mathrm{TN}}(A)=\mathbf{1}^{T} L\left(A, E_{i j}\right) \mathbf{1}$ be the total network sensitivity with respect to the weight $w_{i j}$, where $L\left(A, E_{i j}\right)$ is the Fréchet derivative of $A$ in the direction $E_{i j}$. Consider the addition of one edge to the graph defined by $A$. Then the total network sensitivity is maximized by inserting the edge $e\left(v_{n} \rightarrow v_{1}\right)$. Thus, 


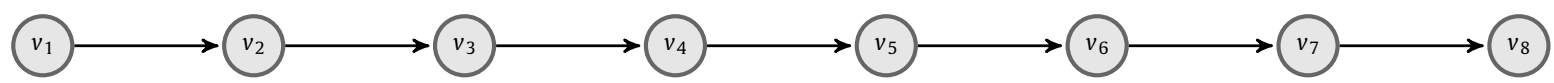

Fig. 3. Graph of Theorem 1 (for $n=8$ ).

$$
\max _{i, j} S_{i j}^{\mathrm{TN}}(A)=S_{n 1}^{\mathrm{TN}}(A)
$$

Proof. Let $r, s$ be integers with $1 \leq r, s \leq n$, and let $\mathbf{e}_{k}=[0, \ldots, 0,1,0, \ldots, 0]^{T} \in \mathbb{R}^{n}$ denotes the $k$ th axis vector and define $E_{r s}=\mathbf{e}_{r} \mathbf{e}_{s}^{T}$. Expanding $L\left(A, E_{r s}\right)$ in terms of powers of $A$ yields

$$
S_{r s}^{\mathrm{TN}}(A)=\mathbf{1}^{T} L\left(A, E_{r s}\right) \mathbf{1}=\mathbf{1}^{T}\left(E_{r s}+\frac{A E_{r s}+E_{r s} A}{2 !}+\frac{A^{2} E_{r s}+A E_{r s} A+E_{r s} A^{2}}{3 !}+\ldots\right) \mathbf{1} .
$$

For notational convenience, denote the sum of the terms in the numerators that contain a total of $k$ powers of $A$ by $H_{r s}^{(k)}$, i.e., $H_{r s}^{(0)}=E_{r s}, H_{r s}^{(1)}=A E_{r s}+E_{r s} A$, etc. Then the right-hand side of (17) can be written as

$$
S_{r s}^{\mathrm{TN}}(A)=\mathbf{1}^{T} L\left(A, E_{r s}\right) \mathbf{1}=\sum_{k=0}^{\infty} \frac{\mathbf{1}^{T} H_{r s}^{(k)} \mathbf{1}}{(k+1) !} .
$$

We will first show that all terms in the sum in (18) except for the $2 n-1$ first ones vanish, and then conclude that $S_{r s}^{\mathrm{TN}}(A)$ is maximized for $r=n$ and $s=1$. First, note that $\mathbf{1}^{T} H_{r s}^{(0)} \mathbf{1}=1$, for all $1 \leq r, s \leq n$. We turn to the expression $\mathbf{1}^{T} H_{r s}^{(1)} \mathbf{1}$ and use the representation $A=\sum_{i=1}^{n-1} \mathbf{e}_{i} \mathbf{e}_{i+1}^{T}$, which yields

$$
H_{r s}^{(1)}=A E_{r s}+E_{r s} A=\sum_{i=1}^{n-1} \mathbf{e}_{i} \mathbf{e}_{i+1}^{T} \mathbf{e}_{r} \mathbf{e}_{s}^{T}+\mathbf{e}_{r} \mathbf{e}_{s}^{T} \sum_{i=1}^{n-1} \mathbf{e}_{i} \mathbf{e}_{i+1}^{T} .
$$

Noting that

$$
\begin{gathered}
\sum_{i=1}^{n-1} \mathbf{e}_{i} \mathbf{e}_{i+1}^{T} \mathbf{e}_{r} \mathbf{e}_{s}^{T}= \begin{cases}\mathbf{e}_{r-1} \mathbf{e}_{s}^{T}, & \text { if } r \geq 2, \\
0, & \text { if } r=1,\end{cases} \\
\mathbf{e}_{r} \mathbf{e}_{s}^{T} \sum_{i=1}^{n-1} \mathbf{e}_{i} \mathbf{e}_{i+1}^{T}= \begin{cases}\mathbf{e}_{r} \mathbf{e}_{s+1}^{T}, & \text { if } s \leq n-1, \\
0, & \text { if } s=n,\end{cases}
\end{gathered}
$$

it follows that $\max _{r, s} \mathbf{1}^{T} H_{r s}^{(1)} \mathbf{1}=2$ is achieved for all $2 \leq r \leq n$ and $1 \leq s \leq n-1$.

We turn to the expression $H_{r s}^{(2)}=A^{2} E_{r s}+A E_{r s} A+E_{r s} A^{2}$ and obtain similarly as above that $\max _{r, s} \mathbf{1}^{T} H_{r s}^{(2)} \mathbf{1}=3$ is achieved for all $3 \leq r \leq n$ and $1 \leq s \leq n-2$. Similarly,

$$
H_{r s}^{(n-1)}=A^{n-1} E_{r s}+A^{n-2} E_{r s} A+A^{n-3} E_{r s} A^{2}+\cdots+E_{r s} A^{n-1}
$$

and $\max _{r, s} \mathbf{1}^{T} H_{r s}^{(n-1)} \mathbf{1}=n$ for $r=n$ and $s=1$. Our findings yield that $\sum_{k=0}^{n-1} \mathbf{1}^{T} H_{r s}^{(k)} \mathbf{1}$ is maximized for $r=n$ and $s=1$. Moreover, $\max _{r, s} \mathbf{1}^{T} H_{r s}^{(k)} \mathbf{1}=k+1$, for $1 \leq k \leq n-1$, i.e., the maximum is the number of terms in the expression for $H_{r s}^{(k)}$.

Now consider matrices $H_{r s}^{(k)}$ for $k \geq n$. Letting $k=n$ yields

$$
H_{r s}^{(n)}=A^{n} E_{r s}+A^{n-1} E_{r s} A+A^{n-2} E_{r s} A^{2}+\cdots+E_{r s} A^{n},
$$

where we observe that

$$
A^{j}=0, \quad j \geq n .
$$

Thus, the expression for $H_{r s}^{(n)}$ has $n-1$ nonvanishing terms and $\max _{r, s} \mathbf{1}^{T} H_{r s}^{n+1} \mathbf{1}=n-1$ is achieved for $r=n$ and $s=1$. For the superscript $2 n-2$, we have

$$
H_{r s}^{(2 n-2)}=A^{2 n-2} E_{r s}+A^{2 n-3} E_{r s} A+\cdots+A^{n-1} E_{r s} A^{n-1}+\cdots+E_{r s} A^{2 n-2} .
$$

Due to (19), the only nonvanishing term in the right-hand side is $A^{n-1} E_{r s} A^{n-1}$, and we obtain

$$
\max _{r, s} \mathbf{1}^{T} H_{r s}^{(2 n-2)} \mathbf{1}=1 .
$$




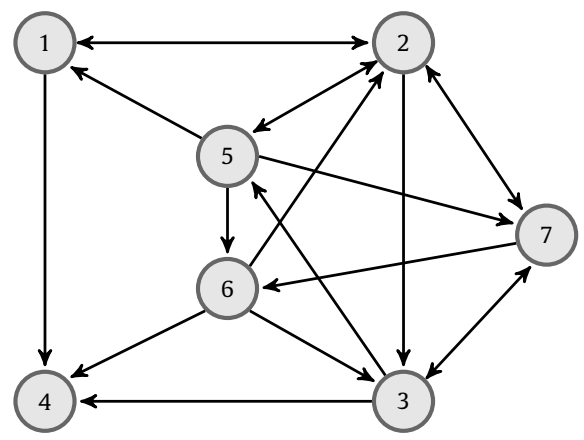

Fig. 4. Graph of Example 3.5.

The maximum is achieved for $r=n$ and $s=1$. We conclude that $\sum_{k=0}^{2 n-2} \mathbf{1}^{T} H_{r s}^{(k)} \mathbf{1}$ is maximized for $r=n$ and $s=1$. Finally, we note that (19) implies that $H_{r s}^{(k)}=0$ for $k \geq 2 n-1$. It follows that $\sum_{k=2 n-1}^{\infty} \mathbf{1}^{T} H_{r s}^{(k)} \mathbf{1}=0$. The above observations show that

$$
\max _{r, s} S_{r s}^{\mathrm{TN}}(A)=\max _{r, s} \sum_{k=0}^{\infty} \frac{\mathbf{1}^{T} H_{r s}^{(k)} \mathbf{1}}{(k+1) !}=\max _{r, s} \sum_{k=0}^{2 n-2} \frac{\mathbf{1}^{T} H_{r s}^{(k)} \mathbf{1}}{(k+1) !}=\sum_{k=0}^{2 n-2} \frac{\mathbf{1}^{T} H_{n 1}^{(k)} \mathbf{1}}{(k+1) !}=S_{n 1}^{\mathrm{TN}}(A),
$$

and the theorem follows.

Example 3.3. Let the graph $\mathcal{G}$ be defined as in Theorem 1 with $n=8$; see Fig. 3. Its total communicability is 11.03 . Consider the graph $\mathcal{G}^{\prime}$ obtained by inserting the edge $e\left(v_{8} \rightarrow v_{1}\right)$. It has total communicability 13.75 . If we instead insert the edge $e\left(v_{1} \rightarrow v_{3}\right)$ in the graph $\mathcal{G}$, we obtain the graph $\mathcal{G}^{\prime \prime}$ with total communicability 12.75 . The difference in total communicability of the graphs $\mathcal{G}^{\prime}$ and $\mathcal{G}^{\prime \prime}$ illustrates that the choice of edge to insert is important when we aim to increase the total communicability as much as possible.

Example 3.4. Consider the undirected unweighted graph obtained by replacing every directed edge in Fig. 3 by an undirected edge and connecting the vertices $v_{1}$ and $v_{8}$ by the undirected edge $e\left(v_{1} \leftrightarrow v_{8}\right)$. We would like to add an undirected edge so that the network communicability is increased the most. For notational convenience, we identify the node $v_{8}$ with $v_{0}$. Due to the circular symmetry of the graph, the total network sensitivities are the same in all directions for which $w_{i j}=0$ and $i \neq j$. Also, the left and right Perron vectors of the adjacency matrix $A$ are the same. Therefore, we cannot determine which edge to add to increase the network communicability the most based on these two approaches. However, the Perron network sensitivities $S_{i, i+4}^{\mathrm{PN}}$ for the most distant nodes (for $i=0,1, \ldots, 4$ ) are the same, and larger than the sensitivities $S_{i j}^{\mathrm{PN}}$ for the nodes $v_{i}$ and $v_{j}$ with $j \neq i+4$. This suggests that to increase the Perron network communicability the most, one should add edges between the most distant nodes.

\subsection{Two other methods to increase or decrease network communicability}

Arrigo and Benzi [2] introduced several methods for the selection of edges to be added to (or removed from) a given directed or undirected graph defined by the adjacency matrix $A$ so as to increase or decrease the network communicability. They define the edge total communicability centrality of an existing edge $e\left(v_{i} \rightarrow v_{j}\right)$ or of a virtual edge $e\left(v_{i}-\rightarrow v_{j}\right)$ as

$$
{ }^{e} T C(i, j)=\left(e^{A} \mathbf{1}\right)_{i}\left(\mathbf{1}^{T} e^{A}\right)_{j} .
$$

They also define another edge total communicability centrality of an existing edge $e\left(v_{i} \rightarrow v_{j}\right)$ or of a virtual edge $e\left(v_{i}-\rightarrow-\right.$ $v_{j}$ ) as

$$
{ }^{e} g T C(i, j)=C_{h}(i) C_{a}(j),
$$

where the total hub communicability of vertex $v_{i}$ and the total authority communicability of vertex $v_{j}$ are given by

$$
C_{h}(i)=\left[U \sinh (\Sigma) V^{T} \mathbf{1}\right]_{i} \text { and } C_{a}(j)=\left[V \sinh (\Sigma) U^{T} \mathbf{1}\right]_{j},
$$

respectively. Here the matrices $U, \Sigma$, and $V$ are the factors of the singular value decomposition $A=U \Sigma V^{T}$.

The following example compares the above approaches to the ones of the present paper.

Example 3.5. Regard the directed unweighted graph shown in Fig. 4. To obtain an irreducible matrix, we use the same procedure as we did in Example 3.2, which gives us $\delta=10^{-5}$ for this example. We would like to add a directed edge 


\section{Table 3}

Example 3.5: The second column lists the edge to be added, and the third and fourth columns show the total network communicability and the Perron network communicability, respectively, when $w_{i j}$ is increased from 0 to 1 .

\begin{tabular}{llll}
\hline Methods & $\{i, j\}$ & $C^{\mathrm{TN}}\left(A+E_{i j}\right)$ & $C^{\mathrm{PN}}\left(\hat{A}+E_{i j}\right)$ \\
\hline${ }^{e} T C(i, j)$ & $\{5,3\}$ & 117.3601 & 92.4046 \\
${ }^{e} g T C(i, j)$ & $\{5,3\}$ & 117.3601 & 92.4046 \\
$S_{i j}^{\mathrm{TN}}$ & $\{7,5\}$ & 127.1123 & 92.4049 \\
$S_{i j}^{\mathrm{PN}}$ & $\{4,5\}$ & 124.1918 & 92.4050 \\
$S_{i j}^{\mathrm{PR}}$ & $\{7,5\}$ & 127.1123 & 92.4049 \\
\hline
\end{tabular}

so that the network communicability is increased as much as possible. To achieve this, the methods by Arrigo and Benzi [2] described above suggest that an edge $e\left(v_{i} \rightarrow v_{j}\right)$ be inserted into the graph so that the index pair $\{i, j\}$ maximizes ${ }^{e} T C(i, j)$ or ${ }^{e} g T C(i, j)$. For the graph of this example, both methods indicate that the edge $e\left(v_{5} \rightarrow v_{3}\right)$ be added to the graph. Table 3 shows the total network communicability and the Perron network communicability after insertion of this edge into the graph. We also evaluate the Perron root sensitivity $\left(S_{i j}^{\mathrm{PR}}\right)$, the total network sensitivity $\left(S_{i j}^{\mathrm{TN}}\right)$, and the Perron network sensitivity $\left(S_{i j}^{\mathrm{PN}}\right)$. The total network sensitivity is seen to be maximal and the Perron root is increased the most for $\{i, j\}=\{7,5\}$, and the Perron network sensitivity is maximized for $\{i, j\}=\{4,5\}$. Table 3 shows the addition of the edges $e\left(v_{7} \rightarrow v_{5}\right)$ or $e\left(v_{4} \rightarrow v_{5}\right)$ to the graph of Fig. 4 to increase the total network communicability and Perron network communicability more than when inserting the edge $e\left(v_{5} \rightarrow v_{3}\right)$. We remark that the selection criteria used in the methods [2] perform well for many graphs, but not for all.

\section{Efficient methods for large-scale networks}

This section discusses some numerical methods for estimating the total network sensitivity, the Perron network sensitivity, and the Perron root sensitivity for large-scale networks. Subsections 4.1, 4.2, and 4.3 describe five iterative Krylov subspace methods to estimate the total network sensitivity. Algorithms for estimating the Perron network sensitivity and the Perron root sensitivity are considered in Subsection 4.4.

\subsection{Applications of the Arnoldi process to large-scale network problems}

The evaluation of the total network sensitivity involves the computation of Fréchet derivatives, which can be done, e.g., by using (6). However, this approach is quite expensive when the adjacency matrix $A$ is large. This section, therefore, describes several iterative Krylov subspace methods to estimate the total network sensitivity. These methods are much cheaper than straightforward evaluation of (6) when the adjacency matrix is large and sparse. Application of $1 \leq m \ll 2 n$ steps of the Arnoldi process to the matrix

$$
M=\left[\begin{array}{cc}
A & E_{i j} \\
0 & A
\end{array}\right] \in \mathbb{R}^{2 n \times 2 n}
$$

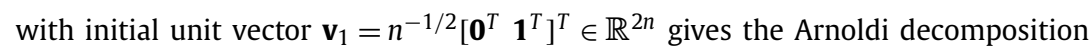

$$
M V_{m}=V_{m} H_{m}+\mathbf{g e}_{m}^{T},
$$

under the assumption that no breakdown occurs. Here, $\mathbf{0}=[0, \ldots, 0]^{T} \in \mathbb{R}^{n}, \mathbf{1}=[1, \ldots, 1]^{T} \in \mathbb{R}^{n}$, and $E_{i j}=\mathbf{e}_{i} \mathbf{e}_{j}^{T} \in \mathbb{R}^{n \times n}$. The matrix $H_{m} \in \mathbb{R}^{m \times m}$ is of upper Hessenberg form with nonvanishing subdiagonal entries. The columns of the matrix $V_{m}=\left[\mathbf{v}_{1}, \mathbf{v}_{2}, \ldots, \mathbf{v}_{m}\right] \in \mathbb{R}^{2 n \times m}$ form an orthonormal basis for the Krylov subspace

$$
\mathcal{K}_{m}\left(M, \mathbf{v}_{1}\right):=\operatorname{span}\left\{\mathbf{v}_{1}, M \mathbf{v}_{1}, \ldots, M^{m-1} \mathbf{v}_{1}\right\},
$$

and $\mathbf{g} \in \mathbb{R}^{2 n}$ is orthogonal to $\mathcal{K}_{m}\left(M, \mathbf{v}_{1}\right)$; see, e.g., Saad [26] for details on the Arnoldi process. Breakdown of the Arnoldi process occurs when a subdiagonal entry of $H_{m}$ vanishes. We will not dwell on this rare situation. It is well known that

$$
p(M) \mathbf{v}_{1}=V_{m} p\left(H_{m}\right) \mathbf{e}_{1},
$$

for all polynomials $p$ of degree at most $m-1$; see, e.g., [25].

We apply the decomposition (21) to compute an approximation of $\mathbf{1}^{T} L\left(A, E_{i j}\right) \mathbf{1}$ using (6) as follows: Define the unit vector $\mathbf{w}=n^{-1 / 2}\left[\mathbf{1}^{T} \mathbf{0}^{T}\right]^{T} \in \mathbb{R}^{2 n}$. Then

$$
\mathbf{1}^{T} L\left(A, E_{i j}\right) \mathbf{1}=n \mathbf{w}^{T} \exp _{0}(M) \mathbf{v}_{1} \approx n \mathbf{w}^{T} V_{m} \exp _{0}\left(H_{m}\right) \mathbf{e}_{1}
$$


Bounds for the discrepancy $\exp _{0}(M) \mathbf{v}_{1}-V_{m} \exp _{0}\left(H_{m}\right) \mathbf{e}_{1}$ can be found in, e.g., [5]. The columns of $V_{m}$ should be reorthogonalized when computed by the Arnoldi process to secure that the vector $\mathbf{w}^{T} V_{m}$ can be evaluated accurately; see [26] for a discussion and implementation of the Arnoldi process with reorthogonalization.

Formula (7) provides an alternative approach to computing an approximation of $\mathbf{1}^{T} L\left(A, E_{i j}\right) \mathbf{1}$ by the Arnoldi process. It follows from (7) that

$$
\lim _{t \rightarrow 0} \frac{\exp _{0}\left(A+t E_{i j}\right)-\exp _{0}(A)}{t}=L\left(A, E_{i j}\right)
$$

which suggests that we apply the Arnoldi process to the matrices $A$ and $A+t E_{i j}$ for some small $t>0$ separately with initial unit vector $\widehat{\mathbf{v}}_{1}=n^{-1 / 2} \mathbf{1} \in \mathbb{R}^{n}$. Thus, application of $m$ steps of the Arnoldi process to $A$ and $A+t E_{i j}$ with initial vector $\widehat{\mathbf{v}}_{1}$ yields the Arnoldi decompositions

$$
A \widehat{V}_{m}=\widehat{V}_{m} \widehat{H}_{m}+\widehat{\mathbf{g}} \mathbf{e}_{m}^{T}, \quad\left(A+t E_{i j}\right) \widetilde{V}_{m}=\widetilde{V}_{m} \widetilde{H}_{m}+\widetilde{\mathbf{g e}}_{m}^{T},
$$

where the columns of $\widehat{V}_{m}$ and $\widetilde{V}_{m}$ form orthonormal bases for the Krylov subspaces $\mathcal{K}_{m}\left(A, \widehat{\mathbf{v}}_{1}\right)$ and $\mathcal{K}_{m}\left(A+t E_{i j}, \widehat{\mathbf{v}}_{1}\right)$, respectively, the matrices $\widehat{H}_{m}, \widetilde{H}_{m} \in \mathbb{R}^{m \times m}$ are of upper Hessenberg form, and the $n$-vectors $\widehat{\mathbf{g}}$ and $\widetilde{\mathbf{g}}$ are orthogonal to the Krylov subspaces $\mathcal{K}_{m}\left(A, \widehat{\mathbf{v}}_{1}\right)$ and $\mathcal{K}_{m}\left(A+t E_{i j}, \widehat{\mathbf{v}}_{1}\right)$, respectively. For some small $t>0$, we use the approximations

$$
L\left(A, E_{i j}\right) \mathbf{1} \approx \frac{\exp _{0}\left(A+t E_{i j}\right)-\exp _{0}(A)}{t} \mathbf{1} \approx n^{1 / 2} \frac{\widetilde{V}_{m} \exp _{0}\left(\widetilde{H}_{m}\right) \mathbf{e}_{1}-\widehat{V}_{m} \exp _{0}\left(\widehat{H}_{m}\right) \mathbf{e}_{1}}{t},
$$

which yield

$$
\mathbf{1}^{T} L\left(A, E_{i j}\right) \mathbf{1} \approx n \frac{\mathbf{e}_{1}^{T} \exp _{0}\left(\widetilde{H}_{m}\right) \mathbf{e}_{1}-\mathbf{e}_{1}^{T} \exp _{0}\left(\widehat{H}_{m}\right) \mathbf{e}_{1}}{t} .
$$

We will illustrate the use of the right-hand sides (23) and (25) in computed examples in Section 5.

\subsection{Applications of the Lanczos biorthogonalization algorithm to large-scale network problems}

We describe how the expression (6) can be approximated by carrying out a few steps of the Lanczos biorthogonalization algorithm [26]. This approach is an alternative to the application of the Arnoldi algorithm described above. Let the vector $\mathbf{v}_{1}$ and matrix $E_{i j}$ be the same as in Subsection 4.1 and define $\mathbf{w}_{1}=\mathbf{v}_{1}$. Application of $1 \leq m \ll 2 n$ steps of the Lanczos biorthogonalization algorithm to the matrix (20) with unit starting vectors $\mathbf{v}_{1}$ and $\mathbf{w}_{1}$ gives, in the absence of breakdown of the recursion formulas, the decompositions

$$
\begin{aligned}
M V_{m} & =V_{m} T_{m}+\mathbf{g}_{1} \mathbf{e}_{m}^{T}, \\
M^{T} W_{m} & =W_{m} T_{m}^{T}+\mathbf{g}_{2} \mathbf{e}_{m}^{T},
\end{aligned}
$$

where the matrix $T_{m} \in \mathbb{R}^{m \times m}$ is tridiagonal and the columns of the matrices $V_{m}=\left[\mathbf{v}_{1}, \mathbf{v}_{2}, \ldots, \mathbf{v}_{m}\right] \in \mathbb{R}^{2 n \times m}$ and $W_{m}=$ $\left[\mathbf{w}_{1}, \mathbf{w}_{2}, \ldots, \mathbf{w}_{m}\right] \in \mathbb{R}^{2 n \times m}$ form a pair of biorthogonal bases for the Krylov subspaces

$$
\begin{aligned}
\mathcal{K}_{m}\left(M, \mathbf{v}_{1}\right) & :=\operatorname{span}\left\{\mathbf{v}_{1}, M \mathbf{v}_{1}, \ldots, M^{m-1} \mathbf{v}_{1}\right\}, \\
\mathcal{K}_{m}\left(M^{T}, \mathbf{w}_{1}\right) & :=\operatorname{span}\left\{\mathbf{w}_{1}, M^{T} \mathbf{w}_{1}, \ldots,\left(M^{T}\right)^{m-1} \mathbf{w}_{1}\right\},
\end{aligned}
$$

respectively. The vectors $\mathbf{g}_{1}, \mathbf{g}_{2} \in \mathbb{R}^{2 n}$ satisfy certain orthogonality relations; see, e.g., Saad [26] for details. Moreover, we have analogously to (22) that

$$
p(M) \mathbf{v}_{1}=V_{m} p\left(T_{m}\right) \mathbf{e}_{1}
$$

for all polynomials $p$ of degree at most $m-1$.

Let $\mathbf{w}=n^{-1 / 2}\left[\mathbf{1}^{T} \mathbf{0}^{T}\right]^{T} \in \mathbb{R}^{2 n}$. Then an approximation of $\mathbf{1}^{T} L\left(A, E_{i j}\right) \mathbf{1}$ analogous to the one determined by application of $m$ steps of the Arnoldi process is given by

$$
\mathbf{1}^{T} L\left(A, E_{i j}\right) \mathbf{1}=n \mathbf{w}^{T} \exp _{0}(M) \mathbf{v}_{1} \approx n \mathbf{w}^{T} V_{m} \exp _{0}\left(T_{m}\right) \mathbf{e}_{1}
$$

To assure that the vector $\mathbf{w}^{T} V_{m}$ can be calculated accurately, the columns of the matrices $V_{m}$ and $W_{m}$ should be rebiorthogonalized when computed by the Lanczos biorthogonalization algorithm; see Parlett et al. [23] for a discussion.

We also will illustrate the following alternative way of using the Lanczos biorthogonalization algorithm to approximate $\mathbf{1}^{T} L\left(A, E_{i j}\right) \mathbf{1}$. Application of $m$ steps of this algorithm to the matrices $A$ and $A+t E_{i j}$ for some small $t>0$ separately with initial unit vectors $\widehat{\mathbf{v}}_{1}=\widehat{\mathbf{w}}_{1}=n^{-1 / 2} \mathbf{1} \in \mathbb{R}^{n}$ yields, assuming that no breakdown occurs, the decompositions

$$
\left\{\begin{array} { l } 
{ A \widehat { V } _ { m } = \widehat { V } _ { m } \widehat { T } _ { m } + \widehat { \mathbf { g } } _ { 1 } \mathbf { e } _ { m } ^ { T } , } \\
{ A ^ { T } \widehat { W } _ { m } = \widehat { W } _ { m } \widehat { T } _ { m } ^ { T } + \widehat { \mathbf { g } } _ { 2 } \mathbf { e } _ { m } ^ { T } , }
\end{array} \quad \text { and } \quad \left\{\begin{array}{l}
\left(A+t E_{i j}\right) \widetilde{V}_{m}=\widetilde{V}_{m} \widetilde{T}_{m}+\widetilde{\mathbf{g}}_{1} \mathbf{e}_{m}^{T}, \\
\left(A+t E_{i j}\right)^{T} \widetilde{W}_{m}=\widetilde{W}_{m} \widetilde{T}_{m}^{T}+\widetilde{\mathbf{g}}_{2} \mathbf{e}_{m}^{T},
\end{array}\right.\right.
$$


where the columns of the matrices $\widehat{V}_{m}$ and $\widehat{W}_{m}$ form a pair of biorthogonal bases for the Krylov subspaces

$$
\begin{aligned}
\mathcal{K}_{m}\left(A, \widehat{\mathbf{v}}_{1}\right) & :=\operatorname{span}\left\{\widehat{\mathbf{v}}_{1}, A \widehat{\mathbf{v}}_{1}, \ldots, A^{m-1} \widehat{\mathbf{v}}_{1}\right\}, \\
\mathcal{K}_{m}\left(A^{T}, \widehat{\mathbf{w}}_{1}\right) & :=\operatorname{span}\left\{\widehat{\mathbf{w}}_{1}, A^{T} \widehat{\mathbf{w}}_{1}, \ldots,\left(A^{T}\right)^{m-1} \widehat{\mathbf{w}}_{1}\right\},
\end{aligned}
$$

respectively, the columns of the matrices $\widetilde{V}_{m}$ and $\widetilde{W}_{m}$ form a pair of biorthogonal bases for the Krylov subspaces

$$
\begin{aligned}
& \mathcal{K}_{m}\left(A+t E_{i j}, \widehat{\mathbf{v}}_{1}\right):=\operatorname{span}\left\{\widehat{\mathbf{v}}_{1},\left(A+t E_{i j}\right) \widehat{\mathbf{v}}_{1}, \ldots,\left(A+t E_{i j}\right)^{m-1} \widehat{\mathbf{v}}_{1}\right\}, \\
& \mathcal{K}_{m}\left(\left(A+t E_{i j}\right)^{T}, \widehat{\mathbf{w}}_{1}\right):=\operatorname{span}\left\{\widehat{\mathbf{w}}_{1},\left(A+t E_{i j}\right)^{T} \widehat{\mathbf{w}}_{1}, \ldots,\left(\left(A+t E_{i j}\right)^{T}\right)^{m-1} \widehat{\mathbf{w}}_{1}\right\},
\end{aligned}
$$

respectively, the matrices $\widehat{T}_{m}, \widetilde{T}_{m} \in \mathbb{R}^{m \times m}$ are tridiagonal, and the vectors $\widehat{\mathbf{g}}_{1}, \widehat{\mathbf{g}}_{2} \in \mathbb{R}^{n}$ satisfy certain orthogonality conditions.

We apply the decompositions (27) similarly as we used the decompositions (24). Thus, for some small $t>0$, we use the approximations

$$
L\left(A, E_{i j}\right) \mathbf{1} \approx \frac{\exp _{0}\left(A+t E_{i j}\right)-\exp _{0}(A)}{t} \mathbf{1} \approx n^{1 / 2} \frac{\widetilde{V}_{m} \exp _{0}\left(\widetilde{T}_{m}\right) \mathbf{e}_{1}-\widehat{V}_{m} \exp _{0}\left(\widehat{T}_{m}\right) \mathbf{e}_{1}}{t},
$$

which yield

$$
\mathbf{1}^{T} L\left(A, E_{i j}\right) \mathbf{1} \approx n \frac{\mathbf{e}_{1}^{T} \exp _{0}\left(\widetilde{T}_{m}\right) \mathbf{e}_{1}-\mathbf{e}_{1}^{T} \exp _{0}\left(\widehat{T}_{m}\right) \mathbf{e}_{1}}{t} .
$$

We will illustrate the use of the right-hand sides (26) and (28) in computed examples in Section 5.

\subsection{Another Arnoldi-based method for approximating the Fréchet derivative}

Kandolf et al. [18] introduced several Krylov subspace methods for approximating the Fréchet derivative. They are defined with the aid of Cauchy integrals and are based on the Lanczos, Arnoldi, and two-sided Arnoldi processes. We outline the Arnoldi-based method; its performance will be illustrated in Section 5.

Consider a directed graph with $n$ nodes and define the associated non-symmetric adjacency matrix $A \in \mathbb{R}^{n \times n}$ and the direction matrix $E=\eta \mathbf{y z} \mathbf{z}^{T} \in \mathbb{R}^{n \times n}$ of rank one. Here $\eta \in \mathbb{R}$ and $\mathbf{y}, \mathbf{z} \in \mathbb{R}^{n}$ are unit vectors. Kandolf et al. [18] described the following approach to approximate the Fréchet derivative of $\exp _{0}(A)$ with respect to the direction $E$.

Application of $1 \leq m \ll n$ steps of the Arnoldi process to the matrices $A$ and $A^{T}$ with initial vectors $\mathbf{y}$ and $\mathbf{z}$, respectively, gives, in the absence of breakdown of the recursion formulas, the Arnoldi decompositions

$$
\begin{aligned}
A V_{m} & =V_{m} G_{m}+\mathbf{g}_{1} \mathbf{e}_{m}^{T}, \\
A^{T} W_{m} & =W_{m} H_{m}+\mathbf{g}_{2} \mathbf{e}_{m}^{T},
\end{aligned}
$$

where the matrices $G_{m}, H_{m} \in \mathbb{R}^{m \times m}$ are of upper Hessenberg form with nonvanishing subdiagonal entries. The columns of the matrices $V_{m}=\left[\mathbf{v}_{1}, \mathbf{v}_{2}, \ldots, \mathbf{v}_{m}\right] \in \mathbb{R}^{n \times m}$ and $W_{m}=\left[\mathbf{w}_{1}, \mathbf{w}_{2}, \ldots, \mathbf{w}_{m}\right] \in \mathbb{R}^{n \times m}$ form orthonormal bases for the Krylov subspaces

$$
\begin{aligned}
\mathcal{K}_{m}(A, \mathbf{y}) & :=\operatorname{span}\left\{\mathbf{y}, A \mathbf{y}, \ldots, A^{m-1} \mathbf{y}\right\}, \\
\mathcal{K}_{m}\left(A^{T}, \mathbf{z}\right) & :=\operatorname{span}\left\{\mathbf{z}, A^{T} \mathbf{z}, \ldots,\left(A^{T}\right)^{m-1} \mathbf{z}\right\},
\end{aligned}
$$

respectively, with $\mathbf{v}_{1}=\mathbf{y}$ and $\mathbf{w}_{1}=\mathbf{z}$. The vectors $\mathbf{g}_{1}, \mathbf{g}_{2} \in \mathbb{R}^{n}$ are orthogonal to $\mathcal{K}_{m}(A, \mathbf{y})$ and $\mathcal{K}_{m}\left(A^{T}, \mathbf{z}\right)$, respectively. Let

$$
B=\left[\begin{array}{cc}
G_{m} & \eta \mathbf{e}_{1} \mathbf{e}_{1}^{T} \\
0 & H_{m}^{T}
\end{array}\right] .
$$

Then the $m$ th Arnoldi approximation of the Fréchet derivative is given by

$$
L_{m}^{\text {Arn }}:=\eta V_{m} X_{m} W_{m}^{T}
$$

where $X_{m}$ can be computed using the equation

$$
\exp _{0}(B)=\left[\begin{array}{cc}
\exp _{0}\left(G_{m}\right) & X_{m} \\
0 & \exp _{0}\left(H_{m}^{T}\right)
\end{array}\right],
$$

and the $m$ th Arnoldi approximation for the total network sensitivity is

$$
\mathbf{1}^{T} L_{m}^{\text {Arn }} \mathbf{1}=\eta \mathbf{1}^{T} V_{m} X_{m} W_{m}^{T} \mathbf{1} ;
$$

see, e.g., Kandolf et al. [18] for details. We refer to this method as the KKRS Arnoldi method in Section 5. 


\section{Table 4}

Example 5.1: The five largest Perron root sensitivities along directions for which $i \neq$ $j$.

\begin{tabular}{ll}
\hline$\{i, j\}$ & $S_{i j}^{\mathrm{PR}}$ \\
\hline$\{248,201\}$ & 0.0471 \\
$\{248,47\}$ & 0.0470 \\
$\{201,47\}$ & 0.0464 \\
$\{248,118\}$ & 0.0452 \\
$\{118,201\}$ & 0.0446 \\
\hline
\end{tabular}

\subsection{Applications of the two-sided Arnoldi and restarted Lanczos methods to large-scale network problems}

The dominant computational burden when studying the Perron root sensitivity and evaluating the quantities $C^{\mathrm{PN}}\left(A+E_{i j}\right)$ and $S_{i j}^{\mathrm{PN}}$ is the calculation of the Perron root and the left and right Perron vectors. For small networks, these quantities easily can be evaluated by using MATLAB functions eig or eigs. For large-scale networks, when the graph $\mathcal{G}$ that determines $A$ is directed, and $A$ therefore is nonsymmetric, these quantities typically can be computed fairly inexpensively by the twosided Arnoldi method, which was introduced by Ruhe [24], and recently has been investigated and improved by Zwaan and Hochstenbach [29]. In the situation when $A$ is symmetric, a restarted Lanczos method, such as [3], can be applied.

\section{Examples with large-scale networks}

This section presents examples with large-scale networks to illustrate the performance of the numerical methods described in Section 4. The computations are carried out using MATLAB R2018b on an Intel Xeon Silver 4116 CPU @ 2.10 GHz (48 cores, 96 threads) equipped with 256 Gbyte RAM. The USAir97 data set used in Example 5.1 can be downloaded from the website [27], the Air500 data set used in Example 5.2 can be downloaded from [1], and the usroads-48 data set used in Example 5.3 can be downloaded from [27].

Define for notational convenience the relative difference

$$
r_{i j}:=\mid(\text { new approximation })-(\text { previous approximation })|/|(\text { previous approximation }) \mid,
$$

where "previous approximation" and "new approximation" denote approximations of the total network sensitivity $S_{i j}^{\mathrm{TN}}$ with respect to the direction $E_{i j}=\mathbf{e}_{i} \mathbf{e}_{j}^{T}$ determined by carrying out $m$ and $m+1$ steps, respectively, of an iterative method.

When applying the methods of Section 4 to estimate the total network sensitivity, we terminate the iterations as soon as $r_{i j}<10^{-4}$ for each $S_{i j}^{\mathrm{TN}}$. We refer to the exact total network sensitivities as the "exact solution", and denote the approximate solutions obtained by using the right-hand sides of eqs. (23), (25), and (29) by the "Arnoldi solution (23)", the "Arnoldi solution (25)", or the "KKRS Arnoldi solution", respectively. Similarly, the approximate solutions determined by the approximations (26) and (28) are referred to as the "Lanczos solution (26)" and the "Lanczos solution (28)", respectively. We let $\eta=1$ in the KKRS Arnoldi method, and $t=2 \cdot 10^{-5}$ in the methods (25) and (28), as well as in the computation of $S_{i j}^{\mathrm{PN}}=\mathbf{1}^{T} L^{\mathrm{PN}}\left(A, E_{i j}\right) \mathbf{1}$. We use the two-sided Arnoldi method to compute the Perron root, and left and right Perron vectors.

Example 5.1. We consider the network USAir97, which is represented by an undirected weighted graph. The graph has 332 nodes, which correspond to American airports in 1997. Undirected edges represent flights from one airport to another and the weight of each undirected edge indicates the frequency of flights between airports. The adjacency matrix $A$ for the graph is irreducible. Our aim is to determine an edge $e\left(v_{i} \leftrightarrow v_{j}\right)$ (or $e\left(v_{i} \leftarrow-\rightarrow v_{j}\right) \notin \mathcal{E}$ ) for $i \neq j$ such that the network communicability is increased the most when increasing the edge-weight $w_{i j}$ and $w_{j i}$ slightly. Thus, to preserve symmetry our perturbations are multiples of $E_{i j}+E_{j i}$ for different $i$ and $j$.

Since the adjacency matrix $A$ is symmetric, we only compute the Perron root sensitivities $S_{i j}^{\mathrm{PR}}$, and explore the total network sensitivity and the Perron network sensitivity to changes in the weights $w_{i j}$ and $w_{j i}$ of edges $e\left(v_{i} \leftrightarrow v_{j}\right.$ ) (or $\left.e\left(v_{i} \leftarrow-\rightarrow v_{j}\right) \notin \mathcal{E}\right)$ with $j>i$ for $i, j \in\{1,2, \ldots, n\}$. Each edge-weight is increased in turn and the quantities $S_{i j}^{\mathrm{TN}}$ and $S_{i j}^{\mathrm{PN}}$ are recorded to find the edge, whose associated weight should be increased. We also evaluate the total network communicability and the Perron network communicability of the graphs obtained when increasing each pair of weights $w_{i j}$ and $w_{j i}$ by one. Table 4 displays the five largest Perron root sensitivities $S_{i j}^{\mathrm{PR}}$ and shows that the Perron root is increased the most when increasing the weight of edge $e\left(v_{248} \leftrightarrow v_{201}\right)$ slightly. The five largest total network sensitivities $S_{i j}^{\mathrm{TN}}$ and Perron network sensitivities $S_{i j}^{\mathrm{PN}}$ with respect to the weight of edge $e\left(v_{i} \leftrightarrow v_{j}\right)$, together with the total network communicability and Perron network communicability attained as the weight of edge $e\left(v_{i} \leftrightarrow v_{j}\right)$ is increased by one are reported in Table 5 . The table shows both the total network communicability and the Perron network communicability to increase the most when the weight of the edge $e\left(v_{118} \leftrightarrow v_{248}\right)$ is increased by one. Since $w_{118,248}=w_{248,118}=0.1733$ this means one should increase the frequency of flights between airport 118 and airport 248 to increase the network communicability with respect to any one edge-weight the most. In this example, since the largest Perron root sensitivities $S^{\mathrm{PR}}(A)_{i, j}$ are very close in 
Table 5

Example 5.1: The five largest total network sensitivities $S_{i j}^{\mathrm{TN}}$ and Perron network sensitivities $S_{i j}^{\mathrm{PN}}$ with respect to changes in the weights of the edge $e\left(v_{i} \leftrightarrow v_{j}\right)$ with $i \neq j$ and $j>i$, as well as the corresponding total network communicability and Perron network communicability when the weight of the edge $e\left(v_{i} \leftrightarrow v_{j}\right)$ is increased by one.

\begin{tabular}{llllll}
\hline$\{i, j\}$ & $S_{i j}^{\mathrm{TN}}$ & $C^{\mathrm{TN}}\left(A+E_{i j}+E_{j i}\right)$ & $\{i, j\}$ & $S_{i j}^{\mathrm{PN}}$ & $C^{\mathrm{PN}}\left(A+E_{i j}+E_{j i}\right)$ \\
\hline$\{118,248\}$ & 408.5090 & 5605.03 & $\{118,248\}$ & 412.30 & 5600.87 \\
$\{47,118\}$ & 402.6977 & 5597.83 & $\{47,118\}$ & 406.85 & 5594.19 \\
$\{118,201\}$ & 401.8136 & 5596.48 & $\{118,201\}$ & 405.79 & 5592.67 \\
$\{118,261\}$ & 396.2688 & 5590.28 & $\{118,261\}$ & 401.14 & 5590.54 \\
$\{67,118\}$ & 386.3636 & 5576.64 & $\{67,118\}$ & 390.32 & 5573.10 \\
\hline
\end{tabular}

\section{Table 6}

CPU time required for evaluating the Perron root sensitivity, the Perron network sensitivity, the exact solution, and the five approximate solutions, as well as the average number of steps $\bar{m}$ demanded by each iterative method to satisfy the stopping criterion.

\begin{tabular}{llll}
\hline Communicability & Method & Average steps & $\begin{array}{l}\text { Elapsed time } \\
\text { (in seconds) }\end{array}$ \\
\hline Perron network communicability & $\begin{array}{l}\text { Perron root sensitivity } \\
\text { Perron network sensitivity }\end{array}$ & $\begin{array}{l}\text { N/A } \\
\text { N/A }\end{array}$ & 0.1 \\
& Exact solution & N/A & 5669 (53 mins) \\
& Arnoldi solution (23) & 9.4774 & 253 \\
Total network $)$ & 4.9942 & 105 \\
& Arnoldi solution (25) & 10.9233 & 1032 \\
& Lanczos solution (26) & 4.9941 & 113 \\
& Lanczos solution (28) & 6.7381 & 226 \\
& KKRS Arnoldi solution & &
\end{tabular}

size, the edge selection furnished by the Perron root sensitivity does not give the largest increase in the Perron network communicability, or the total network communicability.

Table 6 shows the CPU time required for computing the Perron root sensitivity, the Perron network sensitivity, and the total network sensitivity. For the latter, the CPU time for the exact solution and five approximate solutions determined by the Arnoldi and Lanczos biorthogonalization methods is reported. The table also displays the average number of steps needed by the Arnoldi and Lanczos biorthogonalization methods to satisfy the stopping criterion. Both the Perron network sensitivity and the Perron root sensitivity suggest that the same edge-weight be increased, but the computation of the Perron root sensitivity is much cheaper than evaluating the Perron network sensitivity for large networks, as shown in Table 6. Indeed, the Perron root sensitivity may be the only practical indicator of which edge-weight to modify for very large networks. The "exact solution" in the table is evaluated by computing $L\left(A, E_{i j}\right)$ using (6) with $f$ the matrix exponential.

We implemented the Arnoldi method (23) with reorthogonalization to avoid that the computed results are influenced by loss of orthogonality of the Krylov subspace basis. Similarly, rebiorthogonalization is performed for the Lanczos biorthogonalization method (26).

Reducing the tolerance for $r_{i j}$ in (30) to $10^{-5}$ to decide when to terminate the iterations did not change the edges selected when using the Arnoldi-based or Lanczos-based methods.

Fig. 5 shows the exact total network sensitivities and approximate total network sensitivities determined by the five iterative Krylov subspace methods described in Section 4. Specifically, assume that we are interested in evaluating the total network sensitivity $S_{i j}^{\mathrm{TN}}$ for $i \neq j$ and $j>i$ in $\ell$ different directions. We consider increasing one edge-weight of $A$ at each step, then calculate its associated exact (or approximate) total network sensitivity $S_{i j}^{\mathrm{TN}}$, denote it by $s_{1}^{\text {exact }}$ (or $s_{1}^{\text {approx }}$ ), and store it in the vectors $\mathbf{s}^{\text {exact }}=\left[s_{1}^{\text {exact }}\right]$ (or $\mathbf{s}^{\text {approx }}=\left[s_{1}^{a p p r o x}\right]$ ). Repeating this procedure for the $\ell$ directions, we get the vectors $\mathbf{s}^{\text {exact }}=\left[s_{1}^{\text {exact }}, s_{2}^{\text {exact }}, \ldots, s_{\ell}^{\text {exact }}\right]^{T} \in \mathbb{R}^{\ell}$ and $\mathbf{s}^{\text {approx }}=\left[s_{1}^{\text {approx }}, s_{2}^{\text {approx }}, \ldots, s_{\ell}^{\text {approx }}\right]^{T} \in \mathbb{R}^{\ell}$. Each subfigure of Fig. 5 displays the vectors $\boldsymbol{s}^{\text {exact }}$ on the vertical axis and $\mathbf{s}^{\text {approx }}$ for one of the Krylov methods on the horizontal axis. Each pair of vector entries $\left\{s_{i}^{\text {approx }}, s_{i}^{\text {exact }}\right\}$ gives one dot on the graph; if the approximations are accurate, then $s_{i}^{\text {approx }}$ is close to $s_{i}^{\text {exact }}$ and the resulting graph is on a straight line. We conclude that the Arnoldi method (25) is the fastest but the KKRS Arnoldi method is most reliable. The Arnoldi methods demand $\bar{m}=9.4774, \bar{m}=4.9942$, or $\bar{m}=6.7381$ average number of steps to satisfy the stopping criterion, while the Lanczos biorthogonalization methods require 10.9233 or 4.9941 average number of steps. The number of matrix-vector product evaluations needed by the Arnoldi methods (23) and (25) are smaller, since each step only requires one matrix-vector product evaluation, while each step of the KKRS Arnoldi and Lanczos biorthogonalization methods requires two matrix-vector product evaluations, one with $A$ (or $M$ ), and one with $A^{T}$ (or $M^{T}$ ). Each matrixvector product evaluation with $M$ requires two matrix-vector product evaluations with $A$. We conclude from Fig. 5 that the application of the Arnoldi method based on (25) requires the fewest matrix-vector product evaluations followed by the Lanczos biorthogonalization method based on (28). 

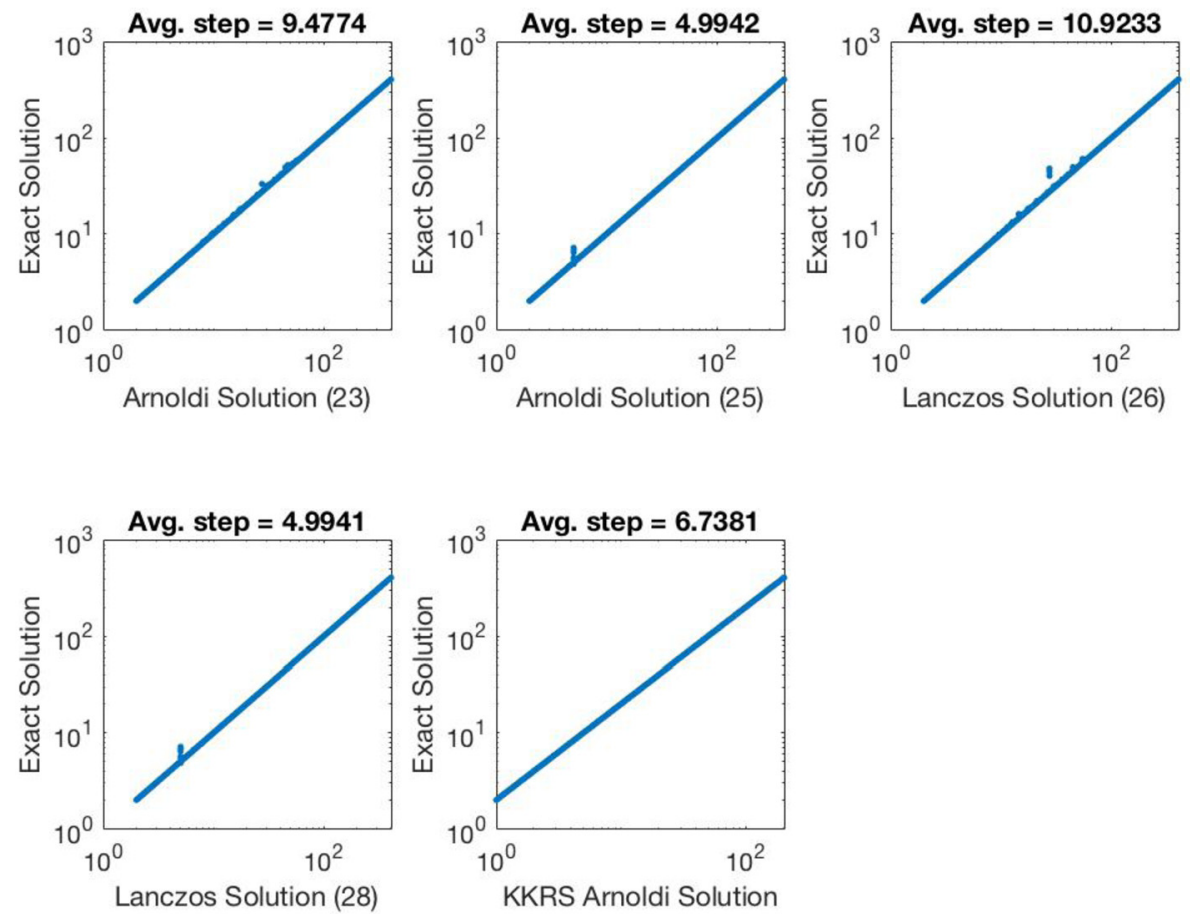

Fig. 5. USAir97: Comparison of the exact and approximate solutions using the five iterative Krylov subspace methods described in Section 4. The heading of each subplot shows the average number of steps $\bar{m}$ required by each iterative method to satisfy the stopping criterion.

Table 7

Example 5.1: The five largest Perron root sensitivities along directions for which $w_{i j}=0$ and $i \neq j$.

\begin{tabular}{ll}
\hline$\{i, j\}$ & $S_{i j}^{\mathrm{PR}}$ \\
\hline$\{224,257\}$ & 0.01341 \\
$\{257,224\}$ & 0.01335 \\
$\{287,224\}$ & 0.01240 \\
$\{261,24\}$ & 0.01230 \\
$\{224,287\}$ & 0.01228 \\
\hline
\end{tabular}

Example 5.2. The network Air500 is represented by an unweighted directed graph with 500 nodes, which model the top 500 airports worldwide based on total passenger volume. Flights are represented by edges; the graph is based on flights within one year from July 1,2007 , to June 30,2008 . The graph is strongly connected. The adjacency matrix associated with the graph therefore is irreducible.

In this example, we want to insert an edge $e\left(v_{i} \rightarrow-v_{j}\right) \notin \mathcal{E}$ that enhances the network communicability the most. We study the Perron root sensitivity by computing the Perron root sensitivity matrix of the adjacency matrix $A$, and investigate the total network sensitivity and the Perron network sensitivity with respect to weights $w_{i j}$, where $w_{i j}=0$ and $i \neq j$. Each edge is added in turn, and the quantities $S_{i j}^{\mathrm{TN}}$ and $S_{i j}^{\mathrm{PN}}$ are recorded to find the optimal edge. We also evaluate the total network communicability and the Perron network communicability of the graph attained when $w_{i j}$ is changed from 0 to 1 .

We recall that the Perron root is increased the most when the Perron root sensitivity $S_{i j}^{\mathrm{PR}}$ is maximized. In this example, we are only interested in adding an edge when $w_{i j}=0$ and $i \neq j$. The five largest Perron root sensitivities $S_{i j}^{\mathrm{PR}}$ of interest are listed in Table 7 . The table indicates that the Perron root is increased the most by inserting the edge $e\left(v_{224} \rightarrow v_{257}\right)$ into the graph. The network communicability also is increased the most by adding this edge as is seen in Table 8 . This table displays the five largest total network sensitivities and Perron network sensitivities with respect to changes in $w_{i j}$, where $w_{i j}=0$ and $i \neq j$, as well as the total network communicability and the Perron network communicability of the graph obtained when changing $w_{i j}$ from 0 to 1 . The table shows both the total network communicability and the Perron network communicability to increase the most by adding an edge with either the largest total network sensitivity $S_{i j}^{\mathrm{TN}}$ or the largest Perron network sensitivity $S_{i j}^{\text {PN }}$. The table suggests that the two busiest airports (224, JFK airport in NY, and 257, LGA airport in NY), be connected. This can be done by adding a shuttle bus between them. 
Table 8

Example 5.2: The five largest total network sensitivities and Perron network sensitivities to changes in $w_{i j}$, where $w_{i j}=0$ and $i \neq j$, together with the corresponding total network communicability and Perron network communicability when $w_{i j}$ is increased from 0 to 1 .

\begin{tabular}{llllll}
\hline$\{i, j\}$ & $S_{i j}^{\mathrm{TN}}$ & $C^{\mathrm{TN}}\left(A+E_{i j}\right)$ & $\{i, j\}$ & $S_{i j}^{\mathrm{PN}}$ & $C^{\mathrm{PN}}\left(A+E_{i j}\right)$ \\
\hline$\{224,257\}$ & $2.5260 \times 10^{36}$ & $1.9418 \times 10^{38}$ & $\{224,257\}$ & $2.5237 \times 10^{36}$ & $1.9386 \times 10^{38}$ \\
$\{257,224\}$ & $2.5161 \times 10^{36}$ & $1.9417 \times 10^{38}$ & $\{257,224\}$ & $2.5103 \times 10^{36}$ & $1.9385 \times 10^{38}$ \\
$\{261,24\}$ & $2.3817 \times 10^{36}$ & $1.9404 \times 10^{38}$ & $\{261,24\}$ & $2.3810 \times 10^{36}$ & $1.9372 \times 10^{38}$ \\
$\{19,124\}$ & $2.3576 \times 10^{36}$ & $1.9401 \times 10^{38}$ & $\{19,124\}$ & $2.3587 \times 10^{36}$ & $1.9369 \times 10^{38}$ \\
$\{24,261\}$ & $2.3529 \times 10^{36}$ & $1.9401 \times 10^{38}$ & $\{24,261\}$ & $2.3457 \times 10^{36}$ & $1.9368 \times 10^{38}$ \\
\hline
\end{tabular}

Table 9

CPU time for the evaluation of the Perron root sensitivity, the Perron network sensitivity, the exact solution, and the five approximated solutions, as well as the average number of steps $\bar{m}$ that each iterative method needed to satisfy the stopping criterion.

\begin{tabular}{llll}
\hline Communicability & Method & Average steps & Elapsed time (in seconds) \\
\hline Perron network communicability & Perron root sensitivity & N/A & 0.65 \\
& Perron network sensitivity & N/A & $9228(2.56 \mathrm{hrs})$ \\
& Exact solution & N/A & $500172(139 \mathrm{hrs})$ \\
& Arnoldi solution (23) & 16.9994 & $2070(34.5 \mathrm{mins})$ \\
Total network communicability & Arnoldi solution (25) & 10.2312 & $1102(18.4 \mathrm{mins})$ \\
& Lanczos solution (26) & 40.1211 & $26381(7.3 \mathrm{hrs})$ \\
& Lanczos solution (28) & 8.0560 & $934(15.6 \mathrm{mins})$ \\
& KKRS Arnoldi solution & 9.9561 & $1521(25.4 \mathrm{mins})$ \\
\hline
\end{tabular}

The CPU time required for computing the Perron root sensitivity, the Perron network sensitivity, the exact solution, and the five approximated solutions, together with the average number of steps that each iterative method carried out, are reported in Table 9. Both the Perron network sensitivity and the Perron root sensitivity suggest the insertion of the same edge to maximize the Perron network communicability, but computing the Perron root sensitivity of $A$ is much cheaper than computing the Perron network sensitivity for large networks, as shown in Table 9. While computing the total network sensitivity, reorthogonalization is carried out for the Arnoldi method (23) and rebiorthogonalization is performed for the Lanczos biorthogonalization method (26).

For this example, the approximations in the stopping criterion (30) are large. Therefore, the Lanczos biorthogonalization method (26) requires many steps to satisfy this criterion when $r_{i j}<10^{-4}$. This makes application of the method expensive. We therefore replaced the matrix $M$ by $M / 3$. Then the Lanczos biorthogonalization method (26) requires fewer steps to satisfy the analogue of (30) with $r_{i j}<10^{-4}$. This modification showed the correct edge to insert. Making the tolerance for $r_{i j}$ smaller than $10^{-4}$ to determine when to terminate the iterative methods did not change the edge selected.

Fig. 6 is analogous to Fig. 5 and shows that the approximate solution obtained using KKRS Arnoldi method to be accurate, followed by the one obtained by Lanczos biorthogonalization method (28), but the latter is faster and requires a smaller average number of steps. The solutions obtained by the Arnoldi method (25) can be seen to be less accurate than those obtained with the KKRS Arnoldi method, and the Lanczos biorthogonalization method (28) (the dots are not as close to a straight line). The solution obtained by the Arnoldi (23) and the Lanczos biorthogonalization methods (26) are the least accurate ones compared to those obtained by the other three iterative methods. On the other hand, each step of the Arnoldi method (23) requires one matrix-vector product evaluation with $M$ (two matrix-vector product evaluations with $A$ ), and each step of the KKRS Arnoldi method demands two matrix-vector product evaluations, one with $A$, and one with $A^{T}$, while each step of the Arnoldi method (25) only requires one matrix-vector product evaluation with $A$. The Lanczos biorthogonalization method (28) is competitive, each step of which requires one matrix-vector product evaluation with $A$ and one with $A^{T}$.

Fig. 7 displays the exact solution and approximate solutions determined by the Arnoldi method (25) by choosing different stopping criteria. The method needs 11.8587 average number of steps (25.66 mins) to satisfy the stopping criterion $r_{i j}<$ $10^{-5}$. The figure shows that the approximation error is smaller when choosing $r_{i j}<10^{-5}$ and the number of matrix-vector product evaluations is still the smallest one. Table 10 displays the airport labels corresponding to the airports of Table 8.

Example 5.3. We consider the network usroads-48, which corresponds to the continental US road network, and is represented by an unweighted undirected graph. The graph has 126146 nodes, which correspond to intersections and road endpoints. Undirected edges represent roads which connect the intersections and endpoints. Our aim is to insert an edge $e\left(v_{i} \leftarrow-\rightarrow v_{j}\right) \notin \mathcal{E}$ such that the network communicability is increased the most. Results of Examples 5.1 and 5.2 show that computing the Perron root sensitivity of the modified adjacency matrix $\hat{A}$ or adjacency matrix $A$ is much cheaper than computing the Perron network sensitivity and total network sensitivity for large networks. Also note that it is not straightforward to determine whether a large network is irreducible. We therefore add a perturbation matrix $\delta A=\delta \cdot \mathbf{1 1}^{T}$ of small norm to the adjacency matrix $A$. This guarantees that the matrix considered is irreducible. To determine the value of $\delta$, we 

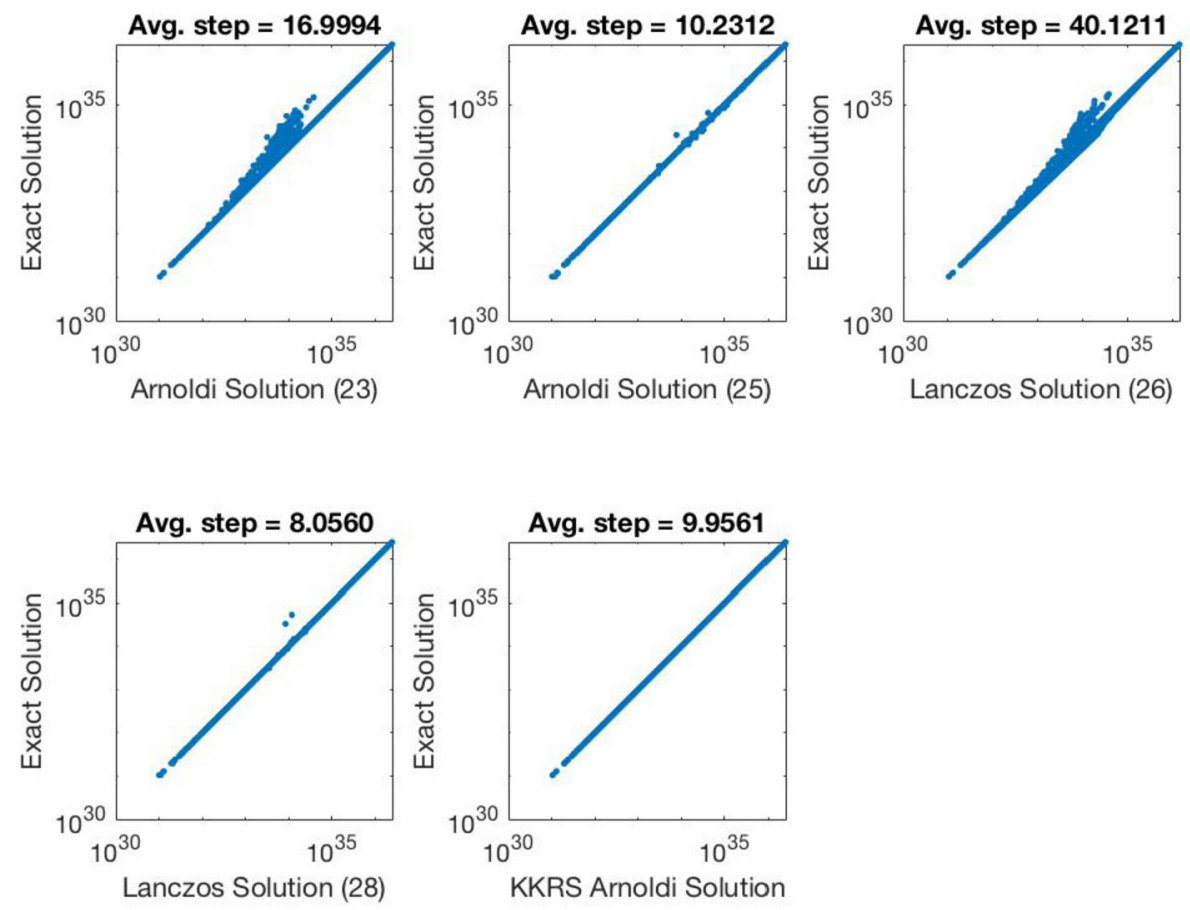

Fig. 6. Air500: Comparison of the exact and approximate solutions using the five iterative Krylov subspace methods described in Section 4 . The heading of each subplot is the average number of steps $\bar{m}$ that each iterative method demands to satisfy the stopping criterion.
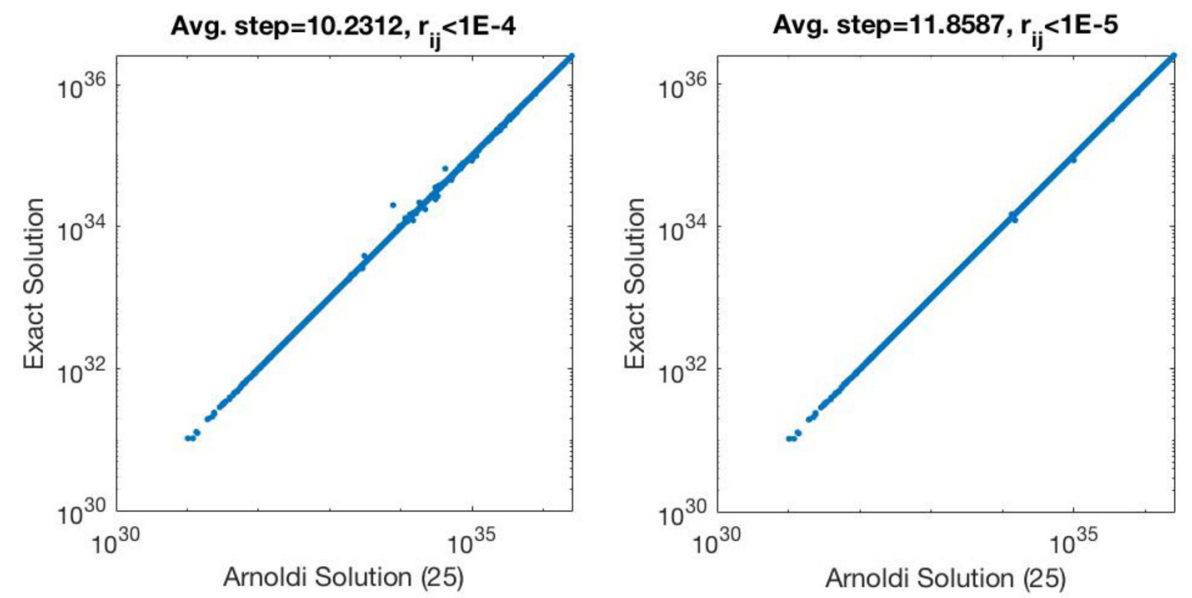

Fig. 7. Air500: Comparison of the exact and approximate solutions using the Arnoldi method (25) by choosing different stopping criterions. The heading of each subplot is the chosen stopping criterion and the average number of steps $\bar{m}$ that the method demands to satisfy the corresponding stopping criterion.

use the same procedure as in Example 3.2. While decreasing the value of $\delta$, we use the available computed Perron vectors for the previous $\delta$-value as initial approximations to compute new Perron vectors associated with the new smaller value of $\delta$. We obtain in this manner the irreducible matrix $\hat{A}=A+\delta A$ with $\delta=10^{-7}$. Note that the matrix $\hat{A}$ is not explicitly formed; we evaluate matrix-vector products with $\hat{A}$ and $\hat{A}^{T}$ without explicitly storing these matrices. This keeps both the storage and computing time low.

Table 11 displays the five largest Perron root sensitivities $S_{i j}^{\mathrm{PR}}$ and shows that the Perron root is increased the most by inserting the edge $e\left(v_{44182} \leftrightarrow v_{44035}\right)$, that is, building a new road between endpoints 44182 and 44035 . The network of the present example is too large to make the evaluation of total network sensitivities and the exact solution practical.

The examples of this section, as well as numerous other numerical experiments, indicate that the Arnoldi-based method (25), the KKRS Arnoldi method, and the Lanczos biorthogonalization method (28) to perform better than the Arnoldi-based method (23) and the Lanczos biorthogonalization method (26). The Arnoldi method (25) typically requires fewer matrix- 
Table 10

\begin{tabular}{ll} 
Airports for airport labels shown in Table 8. \\
\hline Label & Airport \\
\hline 19 & AMS airport in NL \\
24 & ATL airport in GA, USA \\
124 & DFW airport in TX, USA \\
224 & JFK airport in NY, USA \\
257 & LGA airport in NY, USA \\
261 & LHR airport in UK \\
\hline
\end{tabular}

Table 11

Example 5.3: The five largest Perron root sensitivities along directions for which $w_{i j}=0$ and $i \neq j$.

\begin{tabular}{ll}
\hline$\{i, j\}$ & $S_{i j}^{\mathrm{PR}}$ \\
\hline$\{44182,44035\}$ & 0.0898 \\
$\{44067,44323\}$ & 0.0846 \\
$\{44154,44087\}$ & 0.0845 \\
$\{44182,44133\}$ & 0.0797 \\
$\{44182,44294\}$ & 0.0795 \\
\hline
\end{tabular}

vector product evaluations and less computer storage than the KKRS Arnoldi and Lanczos biorthogonalization methods (28). This suggests that the former method may be attractive to use to compute the total network sensitivities $S_{i j}^{\mathrm{TN}}$ when the evaluation of matrix-vector products is very expensive or computer storage is scarce, otherwise the KKRS Arnoldi method should be applied. For large networks, one can compute the Perron root sensitivity. Its computation is much cheaper than the evaluation of the Perron network sensitivity and total network sensitivity. The latter measures therefore are less attractive to use for large networks.

\section{Conclusion}

This paper explores the sensitivity of global communicability measures to small local changes in a network. In particular, we investigate the sensitivity of the network communicability by increasing or decreasing edge-weights, or adding or removing edges such that the total network communicability or Perron network communicability significantly increases or decreases. The latter communicability measure is new and has the advantage of being easy to apply to very large networks. Efficient Krylov subspace-type methods for estimating the total network sensitivity, the Perron root sensitivity, and the Perron network sensitivity are introduced and compared. Computed examples illustrate the feasibility of the methods described.

\section{Acknowledgements}

The authors would like to thank Ernesto Estrada, Marcel Schweitzer, and the referees for comments. Work of ODCC and LR was supported in part by NSF grant DMS-1720259. Work by SN was partially supported by a grant from Sapienza Università di Roma and by INdAM-GNCS.

\section{References}

[1] Air500 Data, https://www.dynamic-connectome.org/?page_id=25.

[2] F. Arrigo, M. Benzi, Edge modification criteria for enhancing the communicability of digraphs, SIAM J. Matrix Anal. Appl. 37 (2016) $443-468$.

[3] J. Baglama, D. Calvetti, L. Reichel, IRBL: an implicitly restarted block Lanczos method for large-scale Hermitian eigenproblems, SIAM J. Sci. Comput. 24 (2003) 1650-1677.

[4] A. Barrat, M. Barthelemy, A. Vespignani, Weighted evolving networks: coupling topology and weight dynamics, Phys. Rev. Lett. 92 (2004) 228701.

[5] B. Beckermann, L. Reichel, Error estimation and evaluation of matrix functions via the Faber transform, SIAM J. Numer. Anal. 47 (2009) $3849-3883$.

[6] M. Benzi, C. Klymko, Total communicability as a centrality measure, J. Complex Netw. 1 (2013) 124-149.

[7] N. Biggs, Algebraic Graph Theory, Cambridge University Press, Cambridge, 1993.

[8] O. De la Cruz Cabrera, M. Matar, L. Reichel, Analysis of directed networks via the matrix exponential, J. Comput. Appl. Math. 355 (2019) 182-192.

[9] O. De la Cruz Cabrera, M. Matar, L. Reichel, Centrality measures for node-weighted networks via line graphs and the matrix exponential, Numer. Algorithms 88 (2021) 583-614.

[10] E. Estrada, The Structure of Complex Networks: Theory and Applications, Oxford University Press, Oxford, 2011.

[11] E. Estrada, Informational cost and networks navigability, Appl. Math. Comput. 397 (2021) 125914

[12] E. Estrada, N. Hatano, Communicability in complex networks, Phys. Rev. E 77 (2008) 036111.

[13] E. Estrada, N. Hatano, M. Benzi, The physics of communicability in complex networks, Phys. Rep. 514 (2012) 89-119.

[14] E. Estrada, D.J. Higham, N. Hatano, Communicability betweenness in complex networks, Physica A 388 (2009) 764-774.

[15] E. Estrada, J.A. Rodriguez-Velazquez, Subgraph centrality in complex networks, Phys. Rev. E 71 (2005) 056103.

[16] N.J. Higham, Functions of Matrices: Theory and Computation, SIAM, Philadelphia, 2008. 
[17] R.A. Horn, C.R. Johnson, Matrix Analysis, Cambridge University Press, Cambridge, 1985.

[18] P. Kandolf, A. Koskela, S.D. Relton, M. Schweitzer, Computing low-rank approximations of the Frèchet derivative of a matrix function using Krylov subspace methods, Numer. Linear Algebra Appl. (2021) e2401.

[19] A. Milanese, J. Sun, T. Nishikawa, Approximating spectral impact of structural perturbations in large networks, Phys. Rev. E 81 (2010) 046112.

[20] M.E.J. Newman, Analysis of weighted networks, Phys. Rev. E 70 (2004) 056131.

[21] M.E.J. Newman, Networks: An Introduction, Oxford University Press, Oxford, 2010.

[22] J. Ortega, W. Rheinboldt, Iterative Solution of Nonlinear Equations in Several Variables, SIAM, Philadelphia, 2000.

[23] B.N. Parlett, D.R. Taylor, Z.A. Liu, A look-ahead Lanczos algorithm for unsymmetric matrices, Math. Comput. 44 (1985) 105-124.

[24] A. Ruhe, The two-sided Arnoldi algorithm for nonsymmetric eigenvalue problems, in: B. Kågström, A. Ruhe (Eds.), Matrix Pencils, Springer, Berlin, 1983, pp. 104-120.

[25] Y. Saad, Analysis of some Krylov subspace approximations to the matrix exponential operator, SIAM J. Numer. Anal. 29 (1992) $209-228$.

[26] Y. Saad, Iterative Methods for Sparse Linear Systems, SIAM, Philadelphia, 2003.

[27] USAir97 data and usroads-48 Data, https://sparse.tamu.edu.

[28] J.H. Wilkinson, Sensitivity of eigenvalues II, Util. Math. 30 (1986) 243-286.

[29] I.N. Zwaan, M.E. Hochstenbach, Krylov-Schur-type restarts for the two-sided Arnoldi method, SIAM J. Matrix Anal. Appl. 38 (2017) $297-321$. 